\title{
TESTS AND COMPARISONS OF VELOCITY-INVERSION TECHNIQUES
}

\author{
B. T. Welsch and W. P. AbBEtT \\ Space Sciences Laboratory, University of California, Berkeley, CA 94720-7450; welsch@ssl.berkeley.edu \\ M. L. DeRosa \\ Lockheed Martin Advanced Technology Center (ADBS/252), 3251 Hanover Street, Palo Alto, CA 94304 \\ G. H. FiSHER \\ Space Sciences Laboratory, University of California, Berkeley, CA 94720-7450 \\ M. K. Georgoulis \\ Applied Physics Laboratory, Johns Hopkins University, 11100 Johns Hopkins Road, Laurel, MD 20723 \\ K. KuSANO \\ Earth Simulator Center, Japan Agency for Marine-Earth Science and Technology, 3173-25 Showa-machi, Kanazawa-ku, Yokohama, \\ Kanagawa 236-0001, Japan \\ D. W. LongCope AND B. RAVINDRA \\ Physics Department, Montana State University, Bozeman, MT 59717-3840 \\ AND \\ P. W. SCHUCK \\ Plasma Physics Division, US Naval Research Laboratory, 4555 Overlook Avenue SW, Washington, DC 20375-0001 \\ Received 2007 March 22; accepted 2007 July 14
}

\begin{abstract}
Recently, several methods that measure the velocity of magnetized plasma from time series of photospheric vector magnetograms have been developed. Velocity fields derived using such techniques can be used both to determine the fluxes of magnetic energy and helicity into the corona, which have important consequences for understanding solar flares, coronal mass ejections, and the solar dynamo, and to drive time-dependent numerical models of coronal magnetic fields. To date, these methods have not been rigorously tested against realistic, simulated data sets, in which the magnetic field evolution and velocities are known. Here we present the results of such tests using several velocity-inversion techniques applied to synthetic magnetogram data sets, generated from anelastic MHD simulations of the upper convection zone with the ANMHD code, in which the velocity field is fully known. Broadly speaking, the MEF, DAVE, FLCT, IM, and ILCT algorithms performed comparably in many categories. While DAVE estimated the magnitude and direction of velocities slightly more accurately than the other methods, MEF's estimates of the fluxes of magnetic energy and helicity were far more accurate than any other method's. Overall, therefore, the MEF algorithm performed best in tests using the ANMHD data set. We note that ANMHD data simulate fully relaxed convection in a high- $\beta$ plasma, and therefore do not realistically model photospheric evolution.
\end{abstract}

Subject headings: MHD — Sun: activity — Sun: magnetic fields — Sun: photosphere

Online material: tar file

\section{WHY STUDY PHOTOSPHERIC VELOCITIES?}

Coronal mass ejections (CMEs) and solar flares are among the primary drivers of space weather disturbances (Gosling 1993), are magnetically driven, and originate in the low solar corona. While measurements of the vector magnetic field in the corona are scarce, the coronal field is strongly coupled to the photospheric magnetic field: because, to a good approximation, flux is frozen to the plasma in the highly conducting corona, the coronal field is "line-tied" to the photosphere and evolves in response to changes in the Sun's photospheric field. Consequently, observations of the magnetic field below the Sun's corona-typically, photospheric or chromospheric magnetograms - provide crucial data to aid in the forecasting and interpretation of space weather events. Although time series of vector magnetograms have historically been rare, NSF's SOLIS (Henney et al. 2002), the Solar Optical Telescope (SOT; Tarbell 2006) on Hinode, and SDO's Helioseismic and Magnetic Imager (HMI; Scherrer et al. 2005) should dramatically improve photospheric vector magnetogram spatial and temporal coverage in the near future.
How can one incorporate time series of magnetograms into forecasting tools? One approach is to analyze changes in the magnetograms' statistical properties and to look for discriminators between active and inactive periods (Leka \& Barnes 2003a, 2003b, 2007). Another approach is to extrapolate a force-free or potential field for each magnetogram in a series and to relate the evolution of the extrapolations' topological structure with CMEs and flares (Luhmann et al. 1998; Barnes et al. 2005), although this method may not show how the coronal field evolves continuously in response to changes in the photospheric field.

Time series of magnetograms can also be used to drive magnetohydrodynamic (MHD) computer simulations of the coronal magnetic field using the observed magnetic field evolution. While theoreticians modeling eruptive events in the corona are free to impose ad hoc velocity and magnetic fields at the model photosphere (Antiochos et al. 1999; Amari et al. 2000; Forbes 2000; Roussev et al. 2004), data-driven simulations require estimates of the actual photospheric velocity field. And although line-ofsight (LOS) magnetic fields can be used for coronal modeling (Lionello et al. 2002), results obtained from LOS fields will not, 
in general, model the actual coronal field, which must satisfy a photospheric boundary condition with specified normal and tangential fields. (Hereafter we refer to tangential fields as horizontal fields to avoid the subscript $t$, which has been used to refer to components of the magnetic field transverse to the LOS and tangential to the solar surface.) Consequently, time series of vector magnetograms, and the velocities derived from such magnetograms, are ideal inputs for data-driven MHD modeling.

How can velocities be derived from vector magnetograms? Chae (2001) applied local correlation tracking (LCT; November \& Simon 1988) to LOS magnetograms to determine the proper motions of magnetic features on the solar surface, and assumed that the inferred flows $\boldsymbol{u}^{\text {(LCT) }}$ were estimates of the horizontal plasma velocities $\boldsymbol{v}_{h}$ (where the $h$ subscript denotes a vector's horizontal components), needed to calculate the flux of magnetic helicity across the photosphere and into the corona. Assuming that the observed evolution of the photospheric magnetic field is coupled to the flow field by the ideal induction equation

$$
\frac{\partial \boldsymbol{B}}{\partial t}=-c(\nabla \times \boldsymbol{E})=\nabla \times(\boldsymbol{v} \times \boldsymbol{B})
$$

Kusano et al. (2002) combined LCT applied to vector magnetograms with the component of equation (1) normal to the magnetogram,

$$
\frac{\partial B_{z}}{\partial t}=\hat{z} \cdot \nabla \times(\boldsymbol{v} \times \boldsymbol{B})=-\nabla \cdot\left(\boldsymbol{v}_{h} B_{z}-v_{z} \boldsymbol{B}_{h}\right),
$$

to derive three-component velocity fields, $v_{x}, v_{y}$, and $v_{z}$, that were "inductive" (Georgoulis \& LaBonte 2006), i.e., consistent with equation (2). Here we have defined the magnetogram surface to be the horizontal plane containing $\hat{\boldsymbol{x}}$ and $\hat{\boldsymbol{y}}$, with a vertical normal $\hat{z}$. As Kusano et al. (2002) noted, only this component of equation (1) is completely specified by vector magnetic field measurements from a single atmospheric layer. The other components of equation (1) contain vertical derivatives of horizontal magnetic field components, and therefore require measurements of the vector magnetic field at a different height in the atmosphere (e.g., the chromosphere), which are only rarely available (Leka \& Metcalf 2003; Metcalf et al. 2005).

Démoulin \& Berger (2003) argued that the observed motions of magnetic flux on the solar photosphere, $\boldsymbol{u}$, result from the combined effects of horizontal plasma velocities transporting vertical magnetic fields and vertical plasma velocities transporting horizontal magnetic fields, via

$$
\boldsymbol{u} B_{z}=\boldsymbol{v}_{h} B_{z}-v_{z} \boldsymbol{B}_{h}
$$

The distinction between apparent motions of flux and plasma velocities led Welsch (2006) to term $\boldsymbol{u}$ the "flux transport velocity." Démoulin \& Berger (2003) suggested that LCT could be used to infer $\boldsymbol{u}$. We note that, in addition to ideal flux transport, diffusive effects can also lead to apparent flux transport velocities (Welsch 2006), although we ignore these effects in the present work.

Since the seminal work of Kusano et al. (2002), still more techniques have been developed that determine velocities from vector magnetograms. Welsch et al. (2004) introduced inductive local correlation tracking (ILCT), in which equation (3) is used to combine LCT results with equation (2) to determine a photospheric flow field. Longcope (2004) developed the minimum-energy fit (MEF), which finds the photospheric velocity field that is strictly consistent with equation (2) and that minimizes the integrated square of the three-component photospheric velocity. Georgoulis
\& LaBonte (2006) extended the minimum-structure method of Georgoulis et al. (2004) to the problem of velocity determination. Schuck (2005) showed that, formally, LCT is not consistent with the induction equation's normal component, which can be expressed as a continuity equation; instead, LCT is consistent with the advection equation. Building on the "differential LCT" method developed by Lucas \& Kanade (1981), Schuck (2006) developed the differential affine velocity estimator (DAVE), which employs least-squares fitting to solve the continuity equation (Schuck 2006). All of the methods listed above can be applied to chromospheric, as well as photospheric, magnetograms.

Velocity estimates derived from magnetograms have applications beyond driving coronal MHD simulations. Analyses by Démoulin \& Berger (2003), Pariat et al. (2005), and Welsch (2006) outline how knowledge of photospheric flow fields can be used to estimate the fluxes of magnetic helicity, energy, and free energy into the corona. Several researchers have used LCT and/or inductive methods to infer flows, and have attempted to correlate derived helicity and energy fluxes with flares and CMEs (Chae 2001; Kusano et al. 2002; Moon et al. 2002; Maeshiro et al. 2005; Régnier et al. 2006).

The recent proliferation of velocity-inversion methods naturally raises the question: Which velocity-inversion method works best? Here we take a first step toward answering this question by testing these routines' abilities to reproduce known flows from MHD simulations of a rising flux rope in the upper solar convection zone, from which we extract "synthetic magnetograms." While these simulations do not accurately model photospheric field evolution, they do provide a valuable tool for testing velocity estimates. The tests presented here are not blind, because such tests would have been inappropriate given the nascent state of the techniques tested. However, blind tests using simulated data that more accurately model photospheric field evolution are planned.

In the next section we describe how we generated the synthetic magnetograms used in our tests. In $\S 3$ we briefly describe each velocity estimation method, and in $\S 4$ we compare the components of each estimated flow field with those of the simulation's flow field, along with quantities derivable from each flow field: electric fields, helicity, and energy fluxes. Finally, in $\S 5$ we summarize our conclusions and discuss their implications.

\section{TEST DATA}

To test the different inversion techniques, we extracted several synthetic magnetograms - two-dimensional horizontal slices of the vector magnetic field - from near the upper boundary of a three-dimensional, Cartesian MHD simulation of a magnetic flux rope rising through a stratified, turbulent model convection zone. Briefly, the nondimensional MHD system of equations is numerically solved in the anelastic limit (see Lantz \& Fan [1999], Fan et al. [1999], and references therein for a description of the anelastic formalism) using the pseudospectral code ANMHD (Fan et al. 1999; Abbett et al. 2000, 2004). The anelastic formulation is an intermediary approximation between a fully compressible treatment and a Boussinesq model, and provides an efficient means of simulating the high- $\beta\left(\beta>10^{4}\right)$ subsurface layers of the solar convection zone over large spatial and temporal scales. However, the anelastic treatment is not well suited to directly modeling surface layers where $\beta \approx 1$ and the acoustic Mach number becomes large; thus, our horizontal slice represents the state of the plasma several thousand kilometers below the visible surface. Consequently, the peak field normal strength in these slices, $\max \left(\left|B_{z}\right|\right) \simeq 7.4 \mathrm{kG}$, exceeds measurements of typical peak photospheric field strengths by a factor of $\sim 3$. Nonetheless, we 

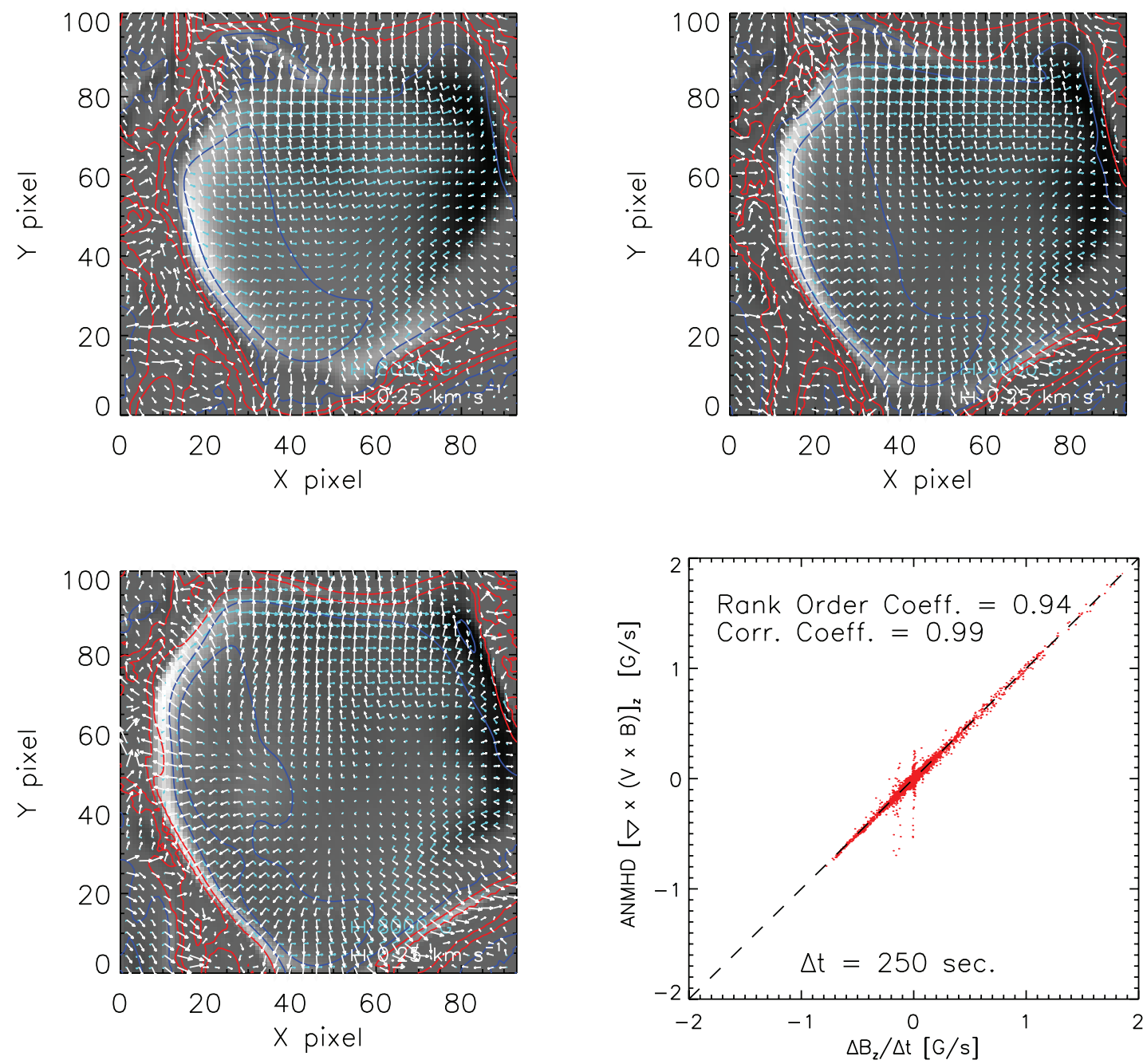

FIG. 1.-Three horizontal slices extracted from the ANMHD run, at $-8361,0$, and $8385 \mathrm{~s}$, are shown in the top left, top right, and bottom left panels. The pixel size is $348 \mathrm{~km}$. The vertical magnetic field is shown in gray scale $\left(\max \left(\left|B_{z}\right|\right)=7.4 \mathrm{kG}\right)$, with the horizontal magnetic field in aqua. Smoothed vertical upflows (downflows) are shown with blue (red) contours, corresponding to 0.12 and $0.04 \mathrm{~km} \mathrm{~s}^{-1}$ upward (downward). (The width of the smoothing window was 3 pixels.) The horizontal velocity field is shown in white. Only components of $\boldsymbol{V}_{\perp}$, the flow perpendicular to $\boldsymbol{B}$, are shown. The perpendicular flows and fields shown in the top right panel are taken as the "true" perpendicular flow and average field in our comparisons. The bottom right panel shows that ANMHD's field evolution obeys the induction equation over the interval $\Delta t=250 \mathrm{~s}$, the shortest time step over which we estimated velocities. This $\Delta t$ is 3 times larger than ANMHD's time step $d t$. Legends in the top left, top right, and bottom left panels show lengths corresponding to $8000 \mathrm{G}$ and $0.25 \mathrm{~km} \mathrm{~s}^{-1}$ for $\boldsymbol{B}_{h}$ and $\boldsymbol{V}_{\perp}$, respectively.

believe that the data set still provides an excellent test bed for our velocity-inversion techniques.

The simulation began with a relatively weak, untwisted, idealized magnetic flux tube, with $B$ less than the parameter $B_{\text {crit }}$ of Fan et al. (2003), inserted into a dynamically and energetically relaxed, field-free, turbulent model convection zone with a resolution of $288 \times 288 \times 72$. The parameter $B_{\text {crit }}$ is the threshold field strength above which flux tubes can maintain their integrity against convection; $B_{\text {crit }} \sim 3 B_{\text {eq }}$, where $B_{\text {eq }}$ is the equipartition field strength at the base of the convection zone. The relevant nondimensional parameters are as follows: The Reynolds number and Prandtl number are defined as $\operatorname{Re} \equiv \rho_{\text {ref }} v_{\text {conv }} H_{\text {ref }} / \mu$ and $\operatorname{Pr} \equiv$ $\mu / \kappa$ and are set to 750 and unity, respectively. Here $\rho_{\text {ref }}$ and $H_{\text {ref }}$ denote the reference density and pressure scale height at the base of the domain, $\kappa$ refers to the coefficient of thermal conductivity, and $\mu$ is the coefficient of dynamic viscosity. The convective velocity is measured in units of $v_{\text {conv }}$, where $v_{\text {conv }} \equiv\left(\delta_{\text {ref }} g H_{\text {ref }}\right)^{1 / 2}$, $\delta_{\text {ref }}$ is the nondimensional superadiabaticity, and $g$ is the constant gravitational acceleration. The magnetic Reynolds number $\operatorname{Re}_{m} \equiv$ $v_{\text {conv }} H_{\text {ref }} / \eta$ is set relatively high, at 3500 . We note that this particular choice of $\mathrm{Re}_{m}$ leads to a certain amount of Fourier ringing in localized strong-field regions near the upper boundary of the simulation domain. Scaled to solar values, the simulation's pixels are $348 \mathrm{~km}$ in extent.

During the flux rope's buoyant ascent toward the surface, the relatively weak magnetic field implied by $B<B_{\text {crit }}$ allows flux to be advected away from the tube's central axis. By the time a significant amount of flux reaches the upper portion of the computational domain, the flux rope has lost its initial cohesion, and much of the magnetic flux has been swept into the downflow lanes between rising convective cells. While the flux rope's spatial extent $(\sim 30 \mathrm{Mm})$ and flux content $\left(\sim 8 \times 10^{21} \mathrm{Mx}\right)$ are on activeregion scales, its spatial distribution is convection-dominated (since $B<B_{\text {crit }}$ ) and more closely resembles the magnetic structure in granulation or supergranulation than that in active regions. To illustrate the evolution of the synthetic magnetograms, we have shown three snapshots of the velocity and magnetic fields in Figure 1.

We extracted seven pairs of magnetograms from data dumps made during the ANMHD run for estimating velocities from the magnetic field evolution between the endpoints of each pair. All pairs were centered on the same time, but the time interval $\Delta t$ 
between each pair's endpoints was chosen to increase approximately logarithmically, with $\Delta t \in\{250,753,1256,2264,4284$, $8374,16,746\} \mathrm{s}$, in units scaled to solar parameters. The smallest $\Delta t$ between data dumps is 3 times larger than ANMHD's time step, $d t$.

The ANMHD velocities and magnetic field were averaged over the shortest time interval to derive the "ground truth" velocity $\boldsymbol{V}_{\perp}$ perpendicular to $\boldsymbol{B}$, used to analyze the accuracy of our methods' estimated velocities. All of our methods assume the magnetic evolution is governed by equation (2). As shown in Figure 1 (bottom right), we verified that the ANMHD flows and field evolution do obey the ideal induction equation, even though $\Delta t \sim$ $3 d t$ for the smallest $\Delta t$. [We also used a similar analysis to confirm that $\partial_{t} B_{z}=-\nabla \cdot\left(\boldsymbol{u} B_{z}\right)$ in the ANMHD simulation, as hypothesized by Démoulin \& Berger (2003). The correlation plot of these quantities (not shown) is essentially identical to that in Fig. 1 (bottom right).] Departures may also arise from using a Fourier method to compute $\nabla \times\left(\boldsymbol{V}_{\perp} \times \boldsymbol{B}\right)$ that differs from ANMHD's method.

Since velocities parallel to $\boldsymbol{B}$ do not affect either the time evolution of the magnetic field or the fluxes of magnetic energy and helicity, we only compare our inferred flows with $\boldsymbol{V}_{\perp}$, the components of the ANMHD velocities perpendicular to $\boldsymbol{B}$,

$$
\boldsymbol{V}_{\perp}=\boldsymbol{V}_{\text {tot }}-\left(\boldsymbol{V}_{\text {tot }} \cdot \boldsymbol{B}\right) \boldsymbol{B} /|\boldsymbol{B}|^{2} .
$$

To enable standardized validation of velocity estimation techniques, we have furnished the data we extracted from the ANMHD run in a tar file in the electronic version of the Astrophysical Journal, so that other velocity estimation methods currently in use, and those developed in the future, can be tested against these benchmark data. The data were archived in several formats: a fits file, IDL's save format, and simple data format (Fisher 2006), ${ }^{1}$ a platform-independent binary format for large data files that preserves numerical accuracy and is readable in C, FORTRAN, and IDL. A README file accompanying each data format describes how the data can be accessed.

\section{VELOCITY DETERMINATION METHODS}

In this section each velocity estimation method that we tested is described briefly, and references to original papers describing the methods in more detail are included. Each method estimates a velocity field whose components will be compared to those of ANMHD's actual flow field in $\S 4$. The first three methods (the LCT code developed by Lockheed Martin Solar and Astrophysics Laboratory [LMSAL], FLCT, and DAVE), presented in chronological order of publication, quantitatively estimate the flux transport velocity, $\boldsymbol{u}$, from magnetogram sequences. The next four methods (the induction method [IM], ILCT, MEF, and minimum structure reconstruction [MSR]), also presented in chronological order of publication, attempt to estimate three-component plasma velocities, $\boldsymbol{v}$, from magnetogram sequences.

\subsection{LMSAL Local Correlation Tracking}

LCT was probably first employed in solar physics by November \& Simon (1988) to determine the proper motions of image features in time series of photospheric white-light images. Title et al. (1995) developed an LCT method that was tuned to solar data, and Hurlburt et al. (1995) subsequently improved it. We present the basic algorithm here, and direct the reader to DeRosa (2001) for additional details.

\footnotetext{
${ }^{1}$ Available for download from http://solarmuri.ssl.berkeley.edu/ fisher/public/ software/SDF/.
}

As input, the algorithm takes two images $I_{1}$ and $I_{2}$ and $N$ points $\boldsymbol{x}_{i}=\left(x_{i}, y_{i}\right)^{T}$ in the image field, where $i=1,2, \ldots, N$, at which to measure the local displacement of features in the two images. For each $\boldsymbol{x}_{i}$ a relative displacement $\delta \boldsymbol{x}_{i}=\left(\delta x_{i}, \delta y_{i}\right)^{T}$ between $I_{1}$ and $I_{2}$ is sought such that the topography of the pixels in the neighborhood of $\boldsymbol{x}_{i}$ in $I_{1}$ best coincides with the topography of the pixels in the neighborhood of $\boldsymbol{x}_{i}+\delta \boldsymbol{x}_{i}$ in $I_{2}$. Mathematically, this amounts to minimizing the merit function,

$$
\begin{aligned}
M\left(\delta \boldsymbol{x}_{i}\right)=\sum_{p=-s}^{+s} \sum_{q=-s}^{+s}\{ & W(p, q)\left[I_{1}\left(x_{i}+p+\frac{\delta x_{i}}{2}, y_{i}+q+\frac{\delta y_{i}}{2}\right)\right. \\
& \left.\left.-I_{2}\left(x_{i}+p-\frac{\delta x_{i}}{2}, y_{i}+q-\frac{\delta y_{i}}{2}\right)\right]^{2}\right\},
\end{aligned}
$$

where the apodization function $W$ weights pixels closer to $\boldsymbol{x}_{i}$ more heavily in the sum, ensuring the optimal $\delta \boldsymbol{x}_{i}$ represents a local displacement. Typically, a two-dimensional Gaussian function with an $e$-folding width of $\sigma$ is used, $W(p, q)=\exp \left[-\left(p^{2}+q^{2}\right) / \sigma^{2}\right]$. The pixel range $[-s, s]$ defines the neighborhood of $\boldsymbol{x}_{i}$, and an $s$ of about $3 \sigma$ works well; choosing a larger $s$ wastes computational time evaluating weakly weighted outlying points. Bilinear interpolation is used to determine the values of $I_{1}$ and $I_{2}$ at offset image coordinates when computing $M$. Accuracy is improved by repeating the minimization, with $I_{1}$ and $I_{2}$ shifted by $\delta \boldsymbol{x}_{i} / 2$ and $-\delta \boldsymbol{x}_{\mathrm{i}} / 2$, respectively, using a higher order, bicubic interpolation scheme, until the match between the shifted $I_{1}$ and $I_{2}$ cannot be improved.

The parameter $\sigma$ in $W$ determines the spatial resolution of the resulting velocity field, $\left\{\delta \boldsymbol{x}_{i} / \Delta t\right\}$. Spatially oversampling the images (spacing the $\left\{\boldsymbol{x}_{i}\right\}$ more closely than $\sigma$ ) often results in smoother flow maps. Here we computed velocities at every pixel, with $\sigma=16$ and $s=3 \sigma$.

As implemented, displacements larger than 1 pixel are interpreted as spurious, and $\delta \boldsymbol{x}_{i}$ is set to 0 ; this means the method performs poorly for large $\Delta t$, when typical displacements exceed a pixel.

\subsection{Fourier Local Correlation Tracking}

Fourier LCT (FLCT; Welsch et al. 2004) employs three distinct, high-level operations to determine a local displacement of features between successive images: masking the initial and final images with a Gaussian windowing function; cross-correlating the two masked images; and finding the peak of the crosscorrelation function. The displacement of this peak from 0 is the inferred spatial displacement of image features in the neighborhood of the windowing function's center.

The correlation function is computed in Fourier space (cf. November \& Simon [1988], who computed the correlation in physical space). A previous version of FLCT interpolated the pixel-scale correlation function onto a finer grid ( 0.02 pixels) and could approximate the peak of the interpolated function to this accuracy. This effectively limited the algorithm's spatial and temporal resolution, requiring tracking over long enough time intervals that typical displacements exceeded this minimum resolution. Our latest version of FLCT, however, estimates the location of the crosscorrelation function's peak by expanding the cross-correlation function in a Taylor series, to second order, in the neighborhood of the peak pixel, an approach also used by J. Chae (2003, private communication). In addition to being more accurate when tracking shorter time intervals, this version of the code is considerably faster.

In applying FLCT to the ANMHD data set, prior experience with FLCT led us to expect to accurately recover velocities only 
in pixels with $\left|B_{z}\right| \gtrsim 370 \mathrm{G}$, or $5 \%$ of $\max \left(\left|B_{z}\right|\right)$. To minimize the impact of threshold-based discontinuities in the inferred flows on ILCT (see below), however, we tracked all pixels with $\left|B_{z}\right|$ above $0.5 \%$ of $\max \left(\left|B_{z}\right|\right)$, or $37 \mathrm{G}$ in scaled units. As in Welsch et al. (2004), we separately tracked positive-flux pixels and negative-flux pixels. We mask with a Gaussian windowing function, $\exp \left(-r^{2} / \sigma^{2}\right)$. We ran trials with the ANMHD data using $\sigma$ of 5,15 , and 25 pixels for the shortest $\Delta t$ and found the best agreement with $\sigma=15$ pixels. We also used $\sigma=15$ pixels for the longer time intervals.

\subsection{Differential Affine Velocity Estimator}

DAVE was proposed as an alternative to LCT for inferring photospheric velocities from LOS magnetograms when information about the vector magnetic field is absent (Schuck $2005,2006)$. This technique implements a variational principle to minimize deviations in the magnitude of the magnetic induction equation. The functional is constrained by the ideal Ohm's law, which relates the flux transport velocity and the electric field, and an affine flux transport velocity profile, which depends linearly on coordinates. The technique is applied to a windowed subregion around each pixel in the magnetogram sequence. The magnetic induction equation is minimized rather than solved exactly to account for deviations from the ideal MHD model, noise, and filling factors (see, e.g., § 3.4 of Démoulin \& Berger 2003).

The variational principle produces an overdetermined system that is solved directly by linear least-squares or total least-squares methods using three frames; two to compute the temporal derivatives and a time-centered third frame for computing the spatial derivatives. For practical applications, three independent frames should be implemented to minimize the correlated noise between the spatial and temporal derivative estimates (Fermuller 2001). The resulting optical flow field and associated uncertainties are statistically consistent with the magnetic induction equation and the affine velocity profile within this aperture.

For the purposes of these tests, an asymmetric window aperture of roughly $20 \times 40$ pixels was implemented. The asymmetric window produced the best performance, probably because of the topto-bottom alignment (in the field of view) of the main magnetic field structures. This window was chosen from the possible window sizes by computing the velocity field for the image pair and then estimating the correlation coefficient between and slope of $d B_{z} / d t$ and $\nabla_{h} \cdot\left(\boldsymbol{u} B_{z}\right)$. The window size with the highest correlation coefficient and a slope of -1 was deemed the best solution. This same objective method for window choice can be applied to observational magnetogram data.

\subsection{The Induction Method}

The induction method (IM), originally developed by Kusano et al. (2002), was the first to explicitly employ the induction equation's normal component, equation (2), to derive photospheric velocities. The method is described in more detail by Kusano et al. (2004), but the basic concepts and solution procedure are briefly reviewed here.

This method is mathematically grounded by the following theorem (Kusano et al. 2002): If the horizontal velocity $\boldsymbol{v}_{h}$ is known, then the vertical velocity $v_{z}$ satisfying equation (2) is uniquely determined as long as the horizontal magnetic field $\boldsymbol{B}_{h}$ possesses at least one null point, where $\boldsymbol{B}_{h}=0$. In active regions, null points of $\boldsymbol{B}_{h}$ usually exist within concentrations of magnetic flux, meaning the uniqueness of $v_{z}$ is often assured.
In this method the vertical velocity $v_{z}$ and the gauge function $\phi$ in the uncurled version of equation (2),

$$
\frac{\partial \boldsymbol{A}_{P}}{\partial t}=\left(\boldsymbol{v}_{h} B_{z}-v_{z} \boldsymbol{B}_{h}\right) \times \hat{z}-\nabla_{h} \phi,
$$

are simultaneously solved by an iterative technique, where $\boldsymbol{A}_{P}$ is the Coulomb-gauge vector potential, with $\hat{z} \cdot \nabla_{h} \times \boldsymbol{A}_{P}=B_{z}$ at $z=0$ and $\nabla_{h} \cdot \boldsymbol{A}_{P}=\hat{z} \cdot \boldsymbol{A}_{P}=0$. Here, $\nabla_{h}=(\partial / \partial x, \partial / \partial y, 0)^{T}$.

By transforming coordinates at a given pixel to a local coordinate system defined by the orthonormal basis

$$
\hat{\boldsymbol{e}}_{1}=\frac{\boldsymbol{B}_{h} \times \boldsymbol{B}_{z}}{\left|\boldsymbol{B}_{h} \times \boldsymbol{B}_{z}\right|}, \quad \hat{\boldsymbol{e}}_{2}=\frac{\boldsymbol{B}_{h}}{\left|\boldsymbol{B}_{h}\right|}, \quad \hat{z},
$$

we can write the component of $\boldsymbol{v}_{h}$ perpendicular to $\boldsymbol{B}_{h}$ as $v_{1}$, and the other component as $v_{2}$. Because the electric fields arising from $v_{1}$ and $v_{z}\left[\left(\boldsymbol{B} \times v_{1} \hat{\boldsymbol{e}}_{1}\right) / c\right.$ and $\left(\boldsymbol{B}_{h} \times v_{z} \hat{z}\right) / c$, respectively $]$ are orthogonal, $v_{1}$ and $v_{z}$ are not coupled. Hence, although the method determines $v_{z}$ consistent with equation (2) to machine accuracy, the electric field parallel to $\boldsymbol{B}_{h}$ (given by $-v_{1} B_{z} / c$ ) must be specified another way, e.g., from LCT. For the tests conducted here, the flux transport velocity $\boldsymbol{u}$ determined by FLCT (as described in $\S 3.2$ ) is input to IM as $\boldsymbol{v}_{h}$. After $v_{z}$ is determined from equation (6), $\boldsymbol{v}_{\perp}$ is derived by subtracting off the component of $\boldsymbol{v}$ parallel to $\boldsymbol{B}$, using $\boldsymbol{v}_{\perp}=\boldsymbol{v}-(\boldsymbol{v} \cdot \boldsymbol{B}) \boldsymbol{B} /|\boldsymbol{B}|^{2}$.

We should note that, since solving equation (6) for $v_{z}$ requires division by $\left|\boldsymbol{B}_{h}\right|$, the solution $v_{z}$ might become singular at points where $\boldsymbol{B}_{h}=0$. The electric field, however, remains well posed even at these points, because it depends on the product $v_{z}\left|\boldsymbol{B}_{h}\right|$. In the results presented here, pixels were not masked in regions where the time-averaged horizontal magnetic field, $\overline{\boldsymbol{B}}_{h}$, vanished.

\subsection{Inductive Local Correlation Tracking}

Welsch et al. (2004) showed that flows derived from FLCT only approximately reproduce the observed evolution in the normal field, $\Delta B_{z} / \Delta t$. To exactly reproduce the observed field evolution, they use a Helmholtz decomposition to express $\boldsymbol{u} B_{z}$ as the sum of the gradient of a scalar function and the curl of a stream function,

$$
\boldsymbol{u} B_{z}=-\nabla_{h} \chi+\nabla_{h} \times \psi \hat{\boldsymbol{z}},
$$

which Longcope (2004) termed the inductive and electrostatic potentials, respectively.

The induction equation gives a Poisson equation for the inductive potential $\chi$, and ILCT uses LCT flows to determine the electrostatic potential $\psi$ by taking the curl of equation (8) and assuming $\boldsymbol{u}=\boldsymbol{u}^{(\mathrm{LCT})}$. Any estimate of $\boldsymbol{u}$ (from, e.g., DAVE) can be used to determine the electrostatic potential in this way. Although $\boldsymbol{u}$ is only estimated in subregions of the entire domain, $\chi$ and $\psi$ are found everywhere. Since ILCT assumes the induction equation only governs the component $\boldsymbol{v}_{\perp}$ of the flow perpendicular to $\boldsymbol{B}$, with

$$
\boldsymbol{v}_{\perp} \cdot \boldsymbol{B}=0,
$$

knowing $\chi$ and $\psi$ means $\boldsymbol{v}_{\perp}$ can be found. The inversion is, however, problematic in regions where $B_{z}$ is weak.

With an estimated $\boldsymbol{u}$, a time-centered $\boldsymbol{B}$, and the change $\Delta B_{z} / \Delta t$ as input, the only other input to ILCT requiring specification is the threshold in $\left|B_{z}\right|$ above which $\boldsymbol{v}_{\perp}$ will be found. To reduce the effects of derivatives of $\chi$ and $\psi$ across the threshold cutoff, we have found that the best approach is to set a very low input threshold ( $1 \mathrm{G}$ here) for computing the potentials, and then filter 
the derived velocities at a higher threshold to exclude velocities derived in regions of weak normal field in postprocessing.

\subsection{The Minimum Energy Fit}

The MEF, a technique to infer the photospheric velocities consistent with magnetic evolution, is described in Longcope (2004). A unique choice is made from the infinity of consistent velocities by minimizing some penalty function. In the present case, we use a penalty function which sums the squares of all photospheric velocity vectors. Such a choice is motivated less by physics than by the desire to find the smallest flow consistent with the observational constraints. This choice of penalty function naturally produces a velocity perpendicular to the local magnetic field (Longcope 2004).

For the present test the MEF technique is applied to the small portion of the vector magnetograms whose magnetic field strength is larger than the defined threshold, $\left|B_{z}\right|=170 \mathrm{G}$. This is done using a mask array which treats all external regions as having zero magnetic field. The time-averaged field $\left(\bar{B}_{z}\right)$ and the difference field $\left(\Delta B_{z} / \Delta t\right)$ are computed from each pair of vector magnetograms. We use a multigrid scheme to speed up the convergence of the iterative MEF algorithm. Vector magnetograms are interpolated to coarser grids, typically one-quarter of the original resolution. The flow fields returned by the MEF are smoothed to remove large velocities near the polarity-inversion lines, caused by poor convergence. These smoothed fields are then fed into the MEF iteration process. This relaxation-and-smoothing process is repeated several times (typically two to three times) until the smallest possible energy is obtained. The resulting inferred flows are then interpolated back to the original vector magnetogram grid.

\subsection{The Minimum Structure Reconstruction Method}

This technique is detailed in Georgoulis \& LaBonte (2006). As with the MEF and ILCT techniques, it employs the inductive and electrostatic potentials, $\chi$ and $\psi$. The inductive potential $\chi$ is determined from equation (2). The electrostatic potential $\psi$ is calculated iteratively by assuming that the vertical component of the velocity $\boldsymbol{v}_{\perp}$ perpendicular to $\boldsymbol{B}$ is zero, $v_{\perp, z}=0$. The technique is general enough to adopt any prescription for $v_{\perp, z}$, so other constraints are possible. The approximation of $v_{\perp, z}=0$ is intended for strongly magnetized layers at photospheric or low chromospheric altitudes and occurs by combining the minimum-structure approximation of Georgoulis et al. (2004) with the additional assumption that the perpendicular velocity $\boldsymbol{v}_{\perp}$ is only governed by perpendicular magnetic gradients $(\nabla B)_{\perp}$ and aims either to sustain or to eliminate them, thus implying $(\nabla B) \times \boldsymbol{B} \cdot \boldsymbol{v}_{\perp}=0$. The MSR approximation simply means that, in the ideal limit, vertical photospheric flows can only occur along the magnetic field lines. Perpendicular vertical flux transport is assumed to be due to nonideal processes (see, e.g., Pariat et al. 2004), and hence cannot be recovered by the ideal induction equation.

For the present study calculations have been performed for regions where $\left|B_{z}\right|>100 \mathrm{G}$ and $\left|\boldsymbol{B}_{h}\right|>200 \mathrm{G}$, to satisfy the strongfield requirement. This combined threshold is usually less strict than the $\left|B_{z}\right|>370 \mathrm{G}$ used for the comparisons between methods in $\S 4$.

Since the ANMHD simulation models the emergence of a buoyant magnetic flux tube in the convection zone, however, $v_{\perp, z} \neq 0$ (Fig. 1). This contradicts MSR's assumption that $v_{\perp, z}=$ 0 , which causes discrepancies between the actual flows and MSR's estimated flows. In particular, the MSR technique produces horizontal velocities larger than ANMHD's to attempt to satisfy the induction equation in the absence of vertical flows.
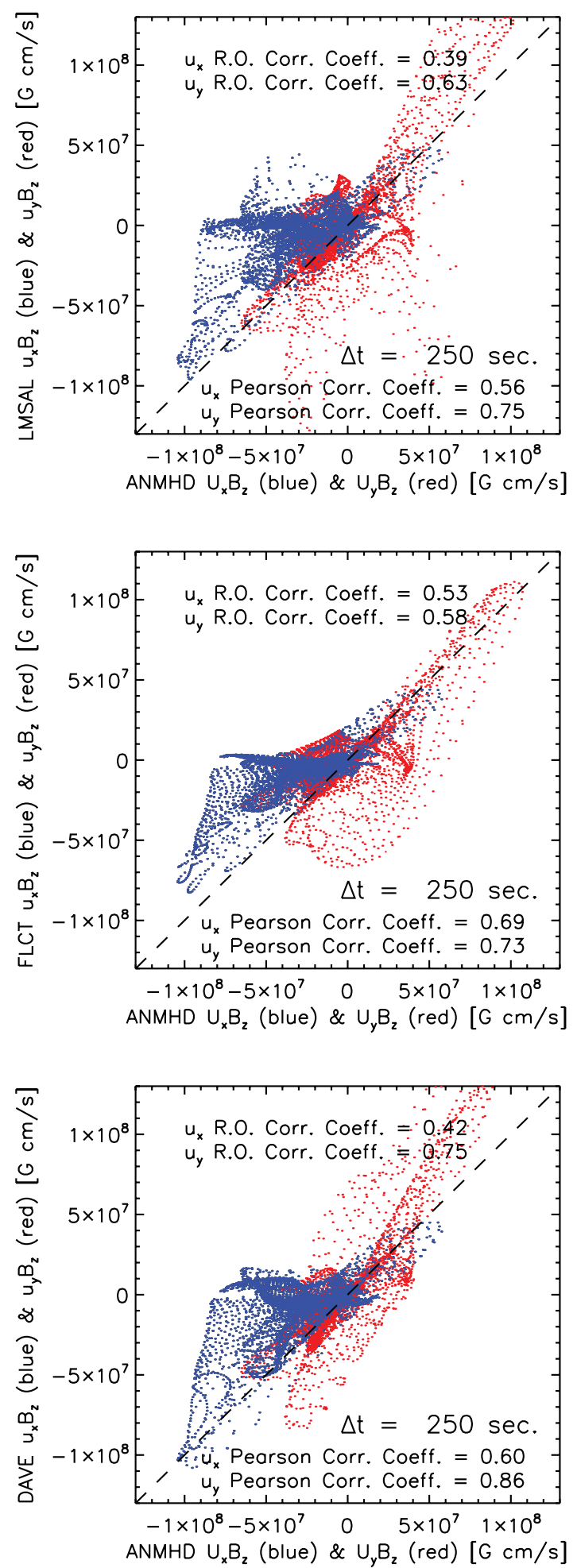

FIG. 2.-Pixel-by-pixel scatter plots of several methods' (LMSAL's LCT, FLCT, and DAVE; top to bottom) estimates of the flux transport rate, $\boldsymbol{u} B_{z}$, defined in eq. (3), with ANMHD's flux transport rate, $\boldsymbol{U} B_{z}$, over the shortest time interval between data dumps, $\Delta t=250 \mathrm{~s}$. Blue and red are used to distinguish $x$ - and $y$-components, respectively. For each method, nonparametric Spearman rank-order correlation coefficients and linear (Pearson) correlation coefficients are shown for both components of the flux transport velocity. 

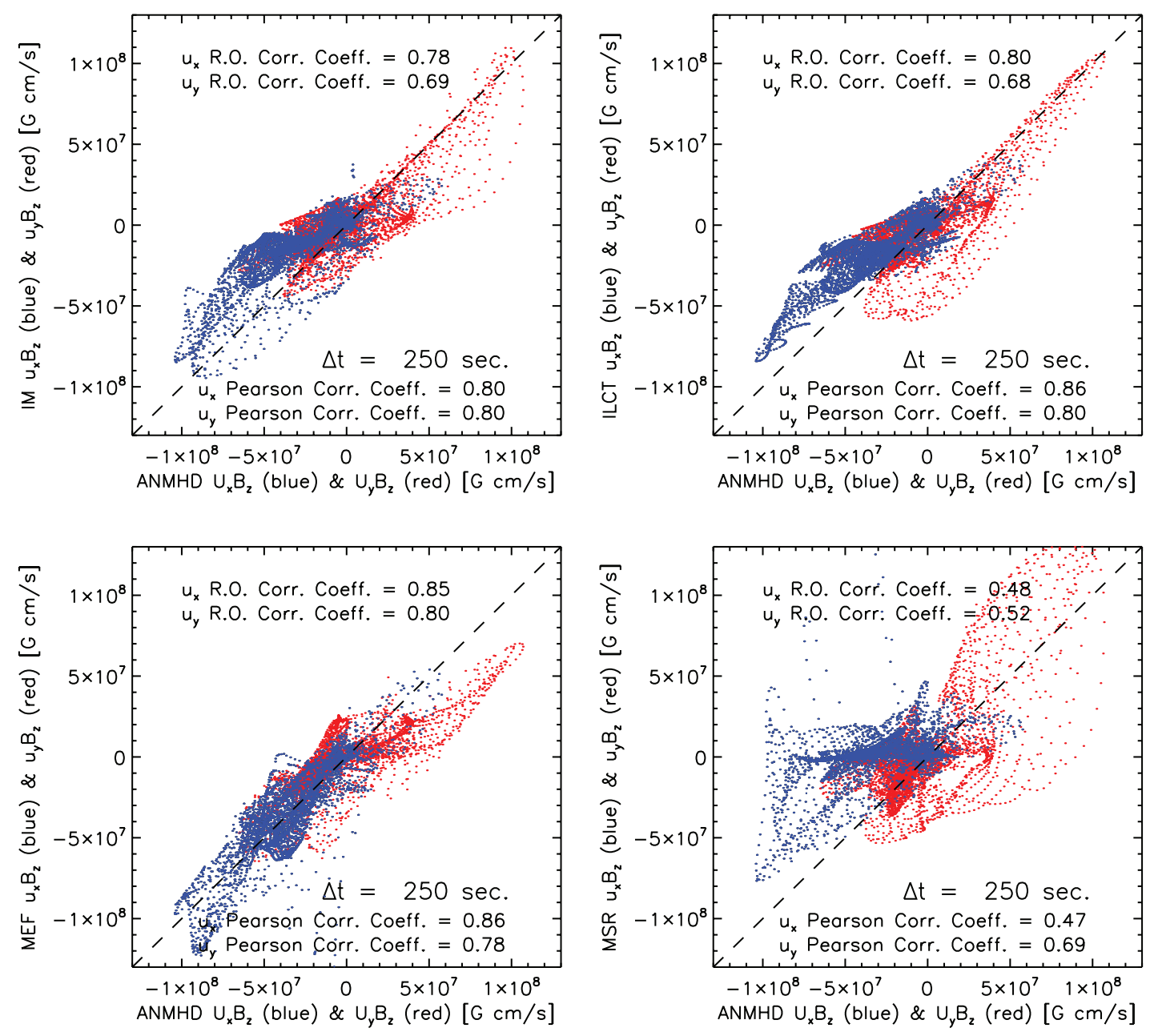

FIG. 3.-As with Fig. 2, but with the remaining methods' (IM, ILCT, MSR, and MEF; clockwise from top left) flux transport estimates.

Simulations that more accurately represent magnetic evolution at the photosphere, where the mass density drops precipitously with increasing altitude, would probably be more appropriate for testing MSR.

\section{RESULTS AND QUANTITATIVE COMPARISONS}

As discussed in $\S 2$, the ANMHD velocities and magnetic field were averaged over the shortest time interval to derive the "ground truth" velocity perpendicular to $\boldsymbol{B}$, used analyzing the accuracy of methods' estimated perpendicular velocities. Here, we compare the components of each method's estimated flow field with those of the ANMHD flow field in several ways. We also compare our accuracy at estimating quantities derivable from flows, including the electric field, $\boldsymbol{E}$, as well as the normal component of its curl; the normal component of the Poynting flux, $S_{z}$; and the flux of magnetic helicity, $d H / d t$, across the magnetogram surface.

For a given time interval, each method only estimated flows in a subset of pixels, usually in regions where $\left|B_{z}\right|$ exceeded a user-imposed threshold. A method's mask is the subset of pixels over which a given method returned a nonzero estimate for the flow. Because of differences in our approaches, the methods' masks differed slightly, but overlapped substantially. Unless otherwise noted, the quantitative comparisons between each method's estimated flows and the ANMHD flows presented below were made using only pixels both that were in that method's mask and in which $\left|B_{z}\right|$ exceeded $5 \%$ of $\max \left(\left|B_{z}\right|\right)$. This threshold level was chosen to mimic solar magnetograms, where peak vertical field strengths are often near $2 \mathrm{kG}$ and thresholds on the order of $100 \mathrm{G}$ have been applied (Welsch et al. 2004; Longcope 2004; Georgoulis \& LaBonte 2006). We varied this threshold and found that the accuracy of the estimates decreases steadily with decreasing threshold. All comparisons contained at least 3700 velocity estimates. (The estimated velocity vectors [white arrows] in Figs. 4 and 5 [see below] convey the areas in which each method's estimates were compared to ANMHD's flows.)

Because the field is precisely known in our simulated magnetograms, each of our velocity estimation methods used one initial and one final magnetogram to estimate the flow field $v$ over the time interval $\Delta t$ between the two. With real magnetographic data, however, averaging the initial and final magnetograms to generate the time-centered magnetogram can propagate correlated noise sources into the resulting velocities (Fermuller 2001). Consequently, for application to real data, using initial, central, and final magnetograms (when available) to determine the time-centered $\boldsymbol{B}$ is preferred over averaging with these methods. Nonetheless, we generally chose to analyze the accuracy of the estimated velocities from the point of view of a researcher who only had two magnetograms, and wished to reconstruct the perpendicular flow at the center of the time interval between them. Hence, the estimated perpendicular flows use only data from the initial and final magnetograms for each $\Delta t$, while the "ground truth" data are derived from the time-averaged velocity and magnetic fields from ANMHD over the shortest $\Delta t$. 
As noted in $\oint 2$, flows parallel to $\boldsymbol{B}$ do not induce a $\Delta B_{z}$, meaning only fluid velocities perpendicular to $\boldsymbol{B}$ can contribute to flows estimated from $\Delta B_{z} / \Delta t$ alone. The flux transport velocity $\boldsymbol{u}$ estimated by DAVE, LMSAL's LCT, and FLCT cannot, therefore, depend on $v_{\|}$, so we can employ equations (3) and (9) to infer $\boldsymbol{v}_{\perp}$ from $\boldsymbol{u}$ for LMSAL's LCT, FLCT, and DAVE via algebraic decomposition (Welsch et al. 2004):

$$
\begin{gathered}
\boldsymbol{v}_{\perp, h}=\boldsymbol{u}-\frac{\boldsymbol{u} \cdot \overline{\boldsymbol{B}}_{h}}{|\overline{\boldsymbol{B}}|^{2}} \overline{\boldsymbol{B}}_{h}, \\
v_{\perp, z}=-\frac{\bar{B}_{z}}{|\overline{\boldsymbol{B}}|^{2}}\left(\boldsymbol{u} \cdot \overline{\boldsymbol{B}}_{h}\right),
\end{gathered}
$$

where $\overline{\boldsymbol{B}}$ is the field averaged between the two magnetograms used to compute $\Delta B_{z}$. Notably, this "perpendicularization" scheme introduces nonzero values for $v_{z}$ in equation (11).

We also applied this perpendicularization to IM and MEF flows. While MEF should tend to minimize $v_{\|}$without any input (e.g., Doppler data) beyond $\Delta B_{z} / \Delta t$, interpolating the MEF flows determined on a coarse grid back to the ANMHD grid introduced parallel flows into some pixels, which had to be removed explicitly. ILCT assumes equation (9) when deriving flows. Velocities derived via MSR do not obey equation (9), since zeroing $v_{\perp, z}$ has the practical effect of introducing parallel flows into regions where $\boldsymbol{v}_{\perp, h} \cdot \boldsymbol{B}_{h} \neq 0$. Nonetheless, we still denote estimated velocities by $\boldsymbol{v}_{\perp}$ to remind the reader that all of our comparisons except those involving MSR include only flows perpendicular to $\boldsymbol{B}$.

\subsection{Flux Transport Velocities}

Generally, only the two-component flux transport velocity, $\boldsymbol{u}$, can be recovered from normal-field magnetograms (or LOS magnetograms, near disk center) alone; some estimate of the horizontal magnetic field is required to determine the three-component fluid velocity, $\boldsymbol{v}_{\perp}$. Some of the methods we investigate (LMSAL's LCT, FLCT, and DAVE) estimate $\boldsymbol{u}$ directly, while three-component velocities $\boldsymbol{v}_{\perp}$ (from ANMHD, IM, ILCT, MEF, and MSR) can be converted to flux transport velocities via equation (3). In Figures 2 and 3 we compare the inferred $\boldsymbol{u} B_{z}$ with ANMHD's flux transport rate, which we denote $\boldsymbol{U} B_{z}$. (We find that dividing by $B_{z}$ to directly compare $\boldsymbol{u}$ with $\boldsymbol{U}$ effectively introduces a weighting that emphasizes results in the weakest field regions, although results from our methods are less accurate in these regions.) Nonparametric Spearman rank-order correlation coefficients and Pearson correlation coefficients (Press et al. 1992, p. 639) are also shown on each plot for each component of the flux transport velocity. We include Spearman coefficients to address the possibilities of lack of linearity between the correlated quantities, departures of errors from the normal distribution, and/or presence of outliers in our estimates.

The statistically significant correlations between the estimated and actual flux transport rates suggest that the methods we have analyzed can quantitatively estimate flux transport rates, as implied by Démoulin \& Berger (2003). The tracking methods (LMSAL's LCT, FLCT, and DAVE), which estimate $\boldsymbol{u}$ directly, do not reconstruct $\boldsymbol{u} B_{z}$ as well as most methods that estimate threecomponent velocities.

\subsection{Comparisons between $\boldsymbol{v}_{\perp}$ and $\boldsymbol{V}_{\perp}$}

To qualitatively compare the estimated and actual velocities, we show in Figures 4 and 5 each method's estimated horizontal velocity vectors, $\boldsymbol{v}_{\perp, h}$ (white arrows), plotted over ANMHD's horizontal velocity vectors, $\boldsymbol{V}_{\perp, h}$ (aqua arrows), with $B_{z}$ shown in gray scale, for the smallest time interval, $\Delta t=250 \mathrm{~s}$.
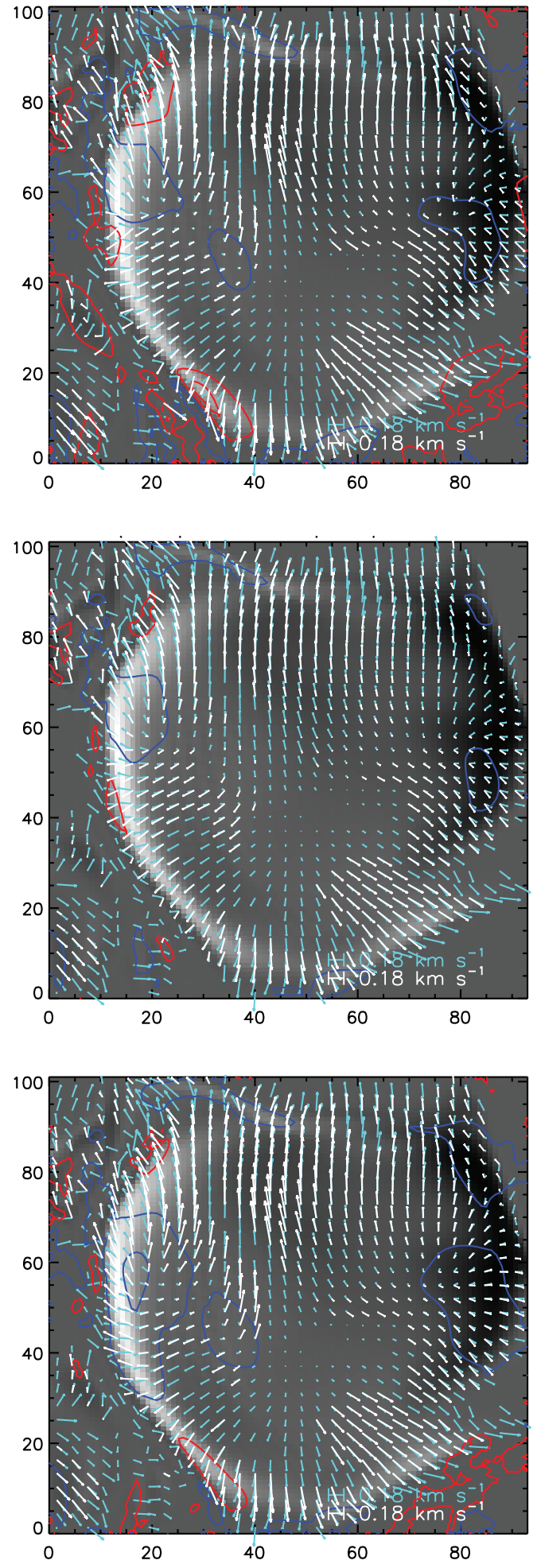

FIG. 4.-Estimated horizontal velocity vectors, $\boldsymbol{v}_{\perp, h}$ (white arrows) plotted over ANMHD's horizontal velocity vectors at $t=0, \boldsymbol{V}_{\perp, h}$ (aqua arrows) for LMSAL's LCT, FLCT, and DAVE (top to bottom), for $\Delta t=250 \mathrm{~s}$. Vectors are shown only for pixels in which $\left|B_{z}\right|>2.5 \%$ of $\max \left(\left|B_{z}\right|\right)$, and for clarity, only every third vector was plotted. Gray scale shows $B_{z}$. Blue (red) contours show smoothed upflows (downflows) corresponding to 0.12 and $0.04 \mathrm{~km} \mathrm{~s}^{-1}$ upward (downward). (The width of the smoothing window was 3 pixels.) 

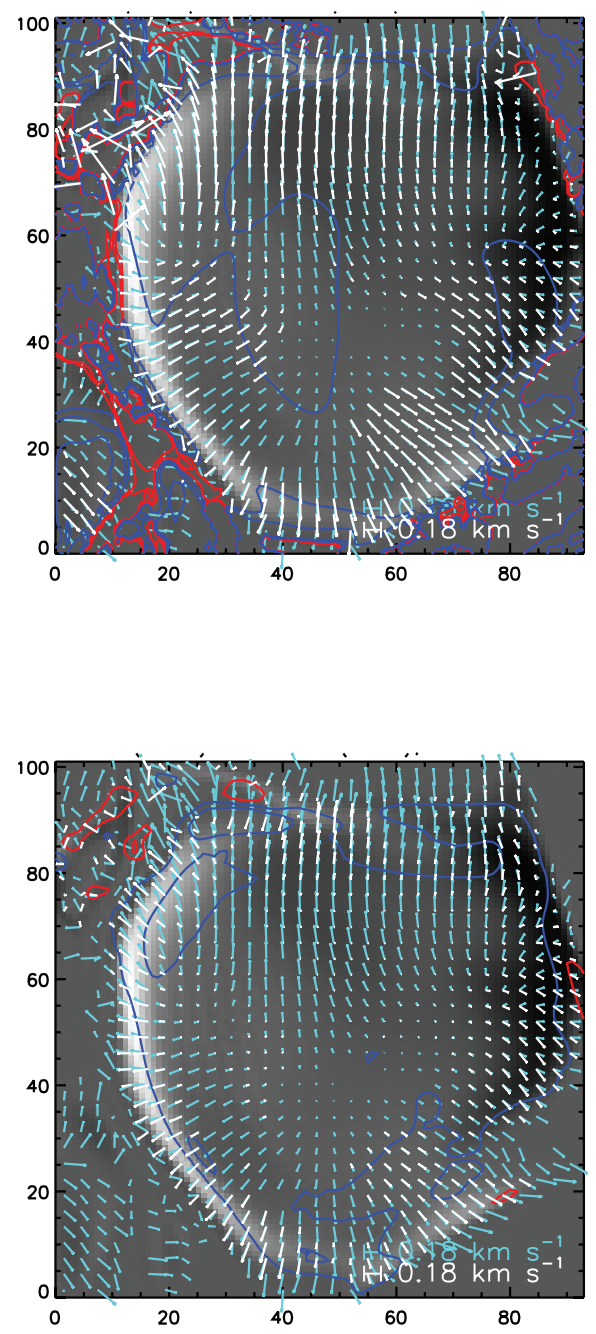
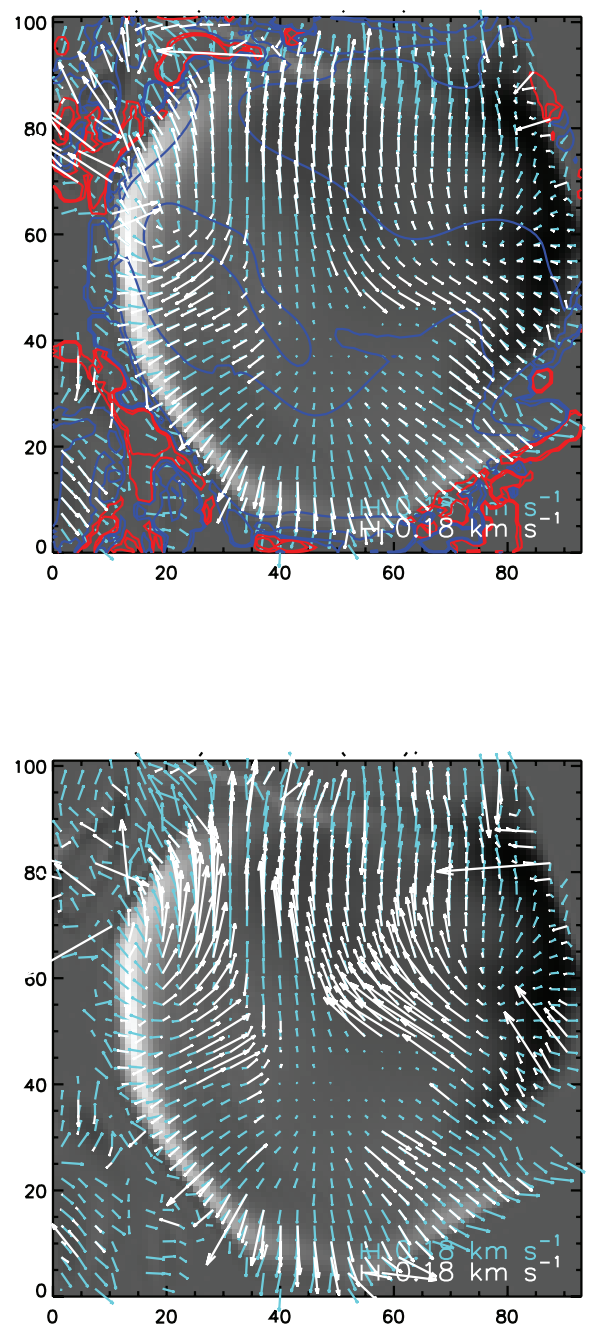

FIG. 5.-As with Fig. 4, but with the remaining methods' (IM, ILCT, MSR, and MEF; clockwise from top left) estimates.

We note that all methods' horizontal velocities become less accurate in weak-field regions near the edges of strong-field features, especially those from ILCT and MSR; this suggests the methods could be improved in such regions. Several of the methods also fare poorly near $(x, y)=(80,50)$, where the horizontal component of $\boldsymbol{V}_{\perp}$ runs parallel to $\boldsymbol{B}_{h}$ (see Fig. 1, top right).

In Figures 6 and 7 we show scatter plots of each method's estimated velocity components with ANMHD's velocity components, again for $\Delta t=250 \mathrm{~s}$. In these plots, red, blue, and black correspond to $v_{x}, v_{y}$, and $v_{z}$, respectively. Each method's plot also shows the nonparametric Spearman rank-order coefficients between that method's estimated velocity components and those of ANMHD.

All of the methods that estimate $V_{\perp, z}$ do so more poorly than they estimate $\boldsymbol{V}_{\perp, h}$, especially the three tracking methods, LMSAL's LCT, FLCT, and DAVE. To investigate why, we considered the theoretical sensitivity of the flux transport velocity, $\boldsymbol{u}$, to vertical and horizontal flows. Since vertical flows should only contribute to the component of $\boldsymbol{u}$ along $\hat{\boldsymbol{e}}_{2}$, defined in equation (7), we took derivatives of the $\hat{\boldsymbol{e}}_{2}$-component of equation (3) with respect to $v_{\perp, z}$ and $v_{2}$, and computed their ratio:

$$
\left|\frac{\partial u_{2} / \partial v_{\perp, z}}{\partial u_{2} / \partial v_{2}}\right|=\left|\frac{\boldsymbol{B}_{h}}{B_{z}}\right| .
$$

This expression implies that the flux transport velocity is less sensitive to vertical flows, relative to horizontal flows, when the magnetic field is more vertical. If the magnetic field in our tests were primarily vertical, then the decreased sensitivity to vertical flows could explain our technique's relatively poor performance at estimating $V_{\perp, z}$. For the pixels compared in Figures 6 and 7, however, the median and mean of the ratio $\left(\left|\boldsymbol{B}_{h}\right| /\left|B_{z}\right|\right)$ are 2.8 and 4.4, respectively, even though $\left|B_{z}\right|$ exceeds $5 \%$ of $\max \left(\left|B_{z}\right|\right)$ in all these pixels. For the same pixels we also compared the mean and median amplitudes of spatial gradients in $B_{h}$ and $B_{z}$, which couple to $\partial_{t} B_{z}$ via $V_{\perp, z}$ and $\boldsymbol{V}_{\perp, h}$, respectively (see eq. [1]), and found that gradients in $B_{h}$ were larger than those in $B_{z}$ by either measure, again implying that our test data should be more sensitive to $V_{\perp, z}$ than to $V_{\perp, h}$. Comparisons of the mean and median of $\left|V_{\perp, z}\right|$ and $\left|\boldsymbol{V}_{\perp, h}\right|$ for the same pixels, however, revealed that horizontal perpendicular flows were twice as large by both measures (a difference of $\sim 1 \sigma$ in $\left|\boldsymbol{V}_{\perp, h}\right|$ and $\sim 2 \sigma$ in $\left|V_{\perp, z}\right|$ ), compared to vertical perpendicular flows. If our methods produce an average absolute error of the same magnitude in both the horizontal and vertical flow components, the fractional error in $V_{\perp, z}$ would exceed that in $\left|\boldsymbol{V}_{\perp, h}\right|$, which could explain the relatively poor estimation of the former.

Closely following the analysis employed by Schrijver et al. (2006) to compare extrapolated magnetic fields with known fields, 

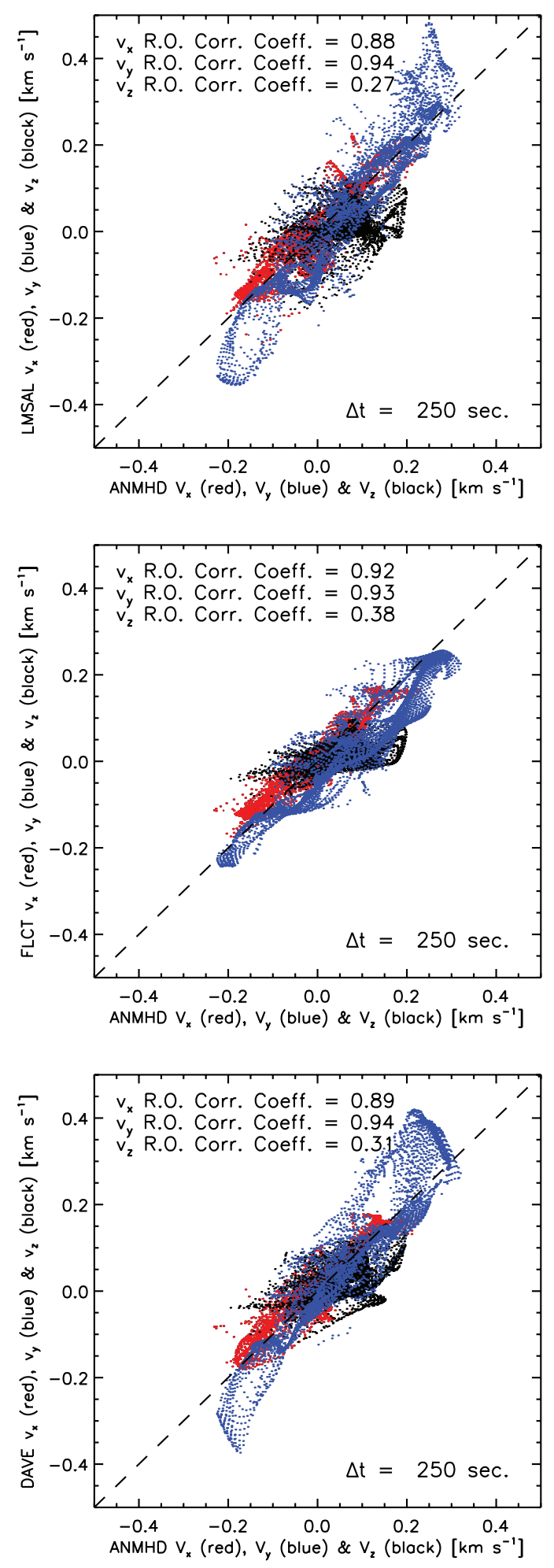

Fig. 6.-Pixel-by-pixel scatter plots comparing several methods' (LMSAL's LCT, FLCT, and DAVE; top to bottom) estimated velocity components with ANMHD's, for $\Delta t=250 \mathrm{~s}$. Red, blue, and black correspond to $v_{x}, v_{y}$, and $v_{z}$, respectively. For each method, nonparametric Spearman rank-order correlation coefficients are also shown for each component of $\boldsymbol{v}_{\perp}$. we quantified the differences between the estimated velocity field, $\boldsymbol{v}_{\perp}$, and the true perpendicular flow field, $\boldsymbol{V}_{\perp}$, in several ways. As a measure of the magnitude of the vector error, we computed the average fractional vector error, $\left\langle\delta \boldsymbol{V}_{\perp}\right\rangle$, defined as

$$
\left\langle\delta \boldsymbol{V}_{\perp}\right\rangle \equiv \frac{1}{N} \sum_{i=1}^{N} \frac{\left|\boldsymbol{V}_{\perp, i}-\boldsymbol{v}_{\perp, i}\right|}{\left|\boldsymbol{V}_{\perp, i}\right|},
$$

where $\boldsymbol{V}_{\perp, i}$ and $\boldsymbol{v}_{\perp, i}$ are the known and estimated velocity vectors at each pixel $i$ for which an algorithm made an estimate, and for which $\left|B_{z}\right|$ exceeded $5 \%$ of $\max \left(\left|B_{z}\right|\right)$; and where $N$ is the total number of pixel estimates used in the comparison. In Figure 8 (top left) we plot each method's $\left\langle\delta \boldsymbol{V}_{\perp}\right\rangle$ as $\Delta t$ increases. To characterize the scatter in the magnitudes of the vector error, we also computed the square root of the variance in the magnitude of the fractional vector error:

$$
\sigma\left(\delta \boldsymbol{V}_{\perp}\right) \equiv \sqrt{\frac{1}{N-1} \sum_{i=1}^{N}\left(\frac{\left|\boldsymbol{V}_{\perp, i}-\boldsymbol{v}_{\perp, i}\right|}{\left|\boldsymbol{V}_{\perp, i}\right|}\right)^{2}} .
$$

In Figure 8 (top right) we plot $\sigma\left(\delta \boldsymbol{V}_{\perp}\right)$ for each method as $\Delta t$ increases. With perfect agreement between $V_{\perp}$ and $\boldsymbol{v}_{\perp}$, both $\left\langle\delta \boldsymbol{V}_{\perp}\right\rangle$ and $\sigma\left(\delta \boldsymbol{V}_{\perp}\right)$ would be zero.

To characterize possible biases in the estimated speeds, we also computed the average fractional error in magnitude, $\langle\delta S\rangle$, where

$$
\langle\delta S\rangle \equiv \frac{1}{N} \sum_{i=1}^{N} \frac{\left|\boldsymbol{v}_{\perp, i}\right|-\left|\boldsymbol{V}_{\perp, i}\right|}{\left|\boldsymbol{V}_{\perp, i}\right|},
$$

and the square root of its variance, $\sigma(\delta S)$, where

$$
\sigma(\delta S) \equiv \sqrt{\frac{1}{N-1} \sum_{i=1}^{N}\left(\frac{\left|\boldsymbol{V}_{\perp, i}\right|-\left|\boldsymbol{v}_{\perp, i}\right|}{\left|\boldsymbol{V}_{\perp, i}\right|}\right)^{2}} .
$$

Perfect agreement between $\left|\boldsymbol{V}_{\perp}\right|$ and $\left|\boldsymbol{v}_{\perp}\right|$ would lead $\langle\delta S\rangle$ and $\sigma(\delta S)$ to vanish. We have plotted $\langle\delta S\rangle$ and $\sigma(\delta S)$ for all the methods in the middle left and middle right panels of Figure 8, respectively, with increasing $\Delta t$. The sign convention for $\langle\delta S\rangle$ is that a negative average speed error corresponds to a bias toward underestimating speeds. FLCT, ILCT, and MEF all clearly underestimate speeds; this is expected for MEF, which minimizes the integrated square of the velocity field, consistent with the evolution of $B_{z}$. Also as expected, MSR overestimates speeds (see $\S 3.7$ ). DAVE consistently estimates speeds most accurately, but has a slight bias toward overestimation, consistent with the findings of Schuck (2006).

We also calculated the vector correlation used by Schrijver et al. (2006), $C_{\mathrm{vec}}$, where

$$
\begin{aligned}
C_{\mathrm{vec}} & \equiv \sum_{i=1}^{N} \boldsymbol{V}_{\perp, i} \cdot \boldsymbol{v}_{\perp, i} /\left(\sum_{i=1}^{N}\left|\boldsymbol{V}_{\perp, i}\right|^{2} \sum_{i=1}^{N}\left|\boldsymbol{v}_{\perp, i}\right|^{2}\right)^{1 / 2} \\
& =\sum_{i=1}^{N}\left|\boldsymbol{V}_{\perp, i}\right|\left|\boldsymbol{v}_{\perp, i}\right| \cos \theta_{i} /\left(\sum_{i=1}^{N}\left|\boldsymbol{V}_{\perp, i}\right|^{2} \sum_{i=1}^{N}\left|\boldsymbol{v}_{\perp, i}\right|^{2}\right)^{1 / 2},
\end{aligned}
$$

where $\theta_{i}$ is the angle between $\boldsymbol{V}_{\perp, i}$ and $\boldsymbol{v}_{\perp, i}$ in the $i$ th pixel. If the vector fields are identical, then $C_{\mathrm{vec}}=1$; if $\boldsymbol{V}_{\perp, i} \perp \boldsymbol{v}_{\perp, i}$, then $C_{\mathrm{vec}}=0$. We have plotted $C_{\mathrm{vec}}$ for all the methods in Figure 8 (bottom left) with increasing $\Delta t$. 

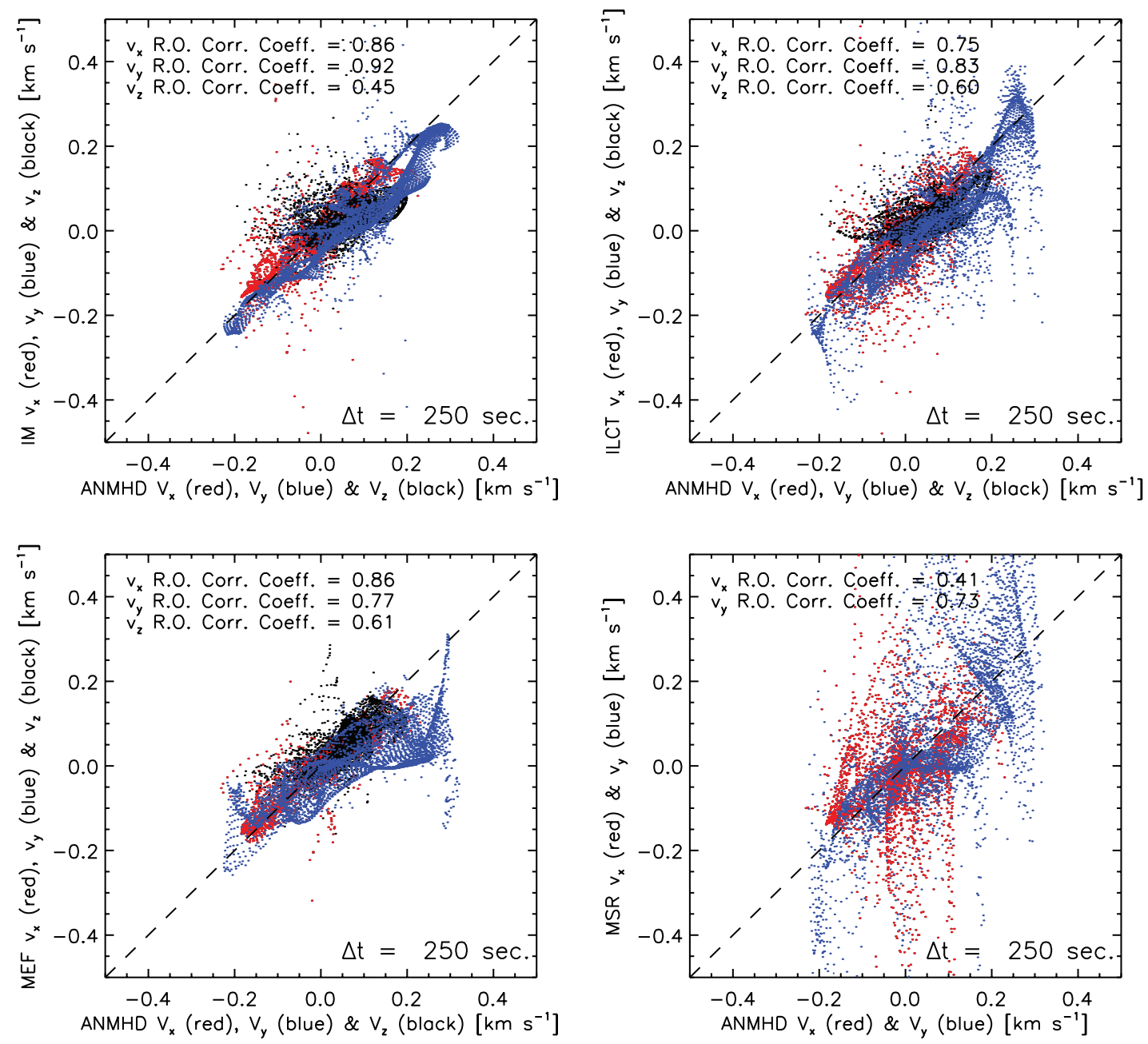

FIG. 7.-As with Fig. 6, but with the remaining methods' (IM, ILCT, MSR, and MEF; clockwise from top left) estimates for each velocity component.

As a measure of the angular differences between $\boldsymbol{V}_{\perp, i}$ and $\boldsymbol{v}_{\perp, i}$, we use the direction correlation, $C_{\mathrm{CS}}$, defined by Schrijver et al. (2006) and based on the Cauchy-Schwarz inequality $(|\boldsymbol{a} \cdot \boldsymbol{b}| \leq|\boldsymbol{a}||\boldsymbol{b}|$ for any two vectors $\boldsymbol{a}$ and $\boldsymbol{b})$ :

$$
C_{\mathrm{CS}} \equiv \frac{1}{N} \sum_{i} \frac{\boldsymbol{V}_{\perp, i} \cdot \boldsymbol{v}_{\perp, i}}{\left|\boldsymbol{V}_{\perp, i}\right|\left|\boldsymbol{v}_{\perp, i}\right|} \equiv \frac{1}{N} \sum_{i} \cos \theta_{i}
$$

When $\boldsymbol{V}_{\perp}$ and $\boldsymbol{v}_{\perp}$ are parallel, $C_{\mathrm{CS}}=1$; when they are antiparallel, $C_{\mathrm{CS}}=-1$; and when they are perpendicular, $C_{\mathrm{CS}}=0$. We have plotted $C_{\mathrm{CS}}$ for all the methods in Figure 8 (bottom right), with increasing $\Delta t$.

Clearly, the codes' performance decreases as the time interval $\Delta t$ between magnetogram pairs increases. Perhaps predictably, significant degradation is apparent for $\Delta t$ much longer than $1900 \mathrm{~s}$, the pixel crossing time, $\tau \sim d x \bar{v}^{-1}$, where $d x=348 \mathrm{~km}$ is the pixel size and $\bar{v}=0.18 \mathrm{~km} \mathrm{~s}^{-1}$ is the average (and median) speed in regions where $\left|B_{z}\right|$ is above $5 \%$ of $\max \left(\left|B_{z}\right|\right)$. For $\Delta t \gg \tau$, typical displacements are larger than a pixel, which can pose problems for methods based on finite-difference schemes with pixelscale spatial derivatives.

Figure 8 also clearly displays where some methods ran into problems. Degradation with increasing $\Delta t$ is clearly present in LMSAL's LCT flows, which results from setting displacements larger than a pixel to 0 . The obvious decrease in accuracy for MEF over the sixth time interval resulted from poor convergence.
All of the methods produce an average fractional vector error greater than 0.5 , much larger than ideal. Considering that most methods estimate speeds to better than $25 \%$ for small $\Delta t$, we surmise that most of the vector error arises from errors in direction. The best direction correlation hovers around 0.866 , corresponding to an average angular error of $30^{\circ}$; two equal-length vectors pointing $30^{\circ}$ apart have a fractional vector difference of 0.52 , right about the average fractional vector error. To characterize the null hypothesis, we compute the average fractional vector error for a distribution of randomly directed but equal-length vectors, $\left\langle\delta \boldsymbol{V}_{\text {rand }}\right\rangle$,

$$
\left\langle\delta \boldsymbol{V}_{\text {rand }}\right\rangle=\int_{0}^{2 \pi} d \theta^{\prime} \sqrt{2-2 \cos \theta^{\prime}} / \int_{0}^{2 \pi} d \theta^{\prime}=4 / \pi \simeq 1.3 .
$$

The statistical analyses presented in Figure 8 were all computed by comparing the three-component estimated velocities $\boldsymbol{v}_{\perp}$ with velocities $\boldsymbol{V}_{\perp}$ from ANMHD, even though some of the estimation methods were developed to infer the flux transport velocity, $\boldsymbol{u}$. We used similar comparisons to analyze the methods' performance at estimating $\boldsymbol{U} B_{z}$. Consistent with the lower correlation coefficients for components of $\boldsymbol{U} B_{z}$ compared to components of $\boldsymbol{V}_{\perp}$, we found that most methods did worse at estimating both the magnitude and the direction of $\boldsymbol{U} B_{z}$ than of $\boldsymbol{V}_{\perp}$ : most methods' fractional vector error increased by 0.2 , and most methods exhibited a negative bias in magnitude of 0.25 . Both MEF and IM, however, did improve: 

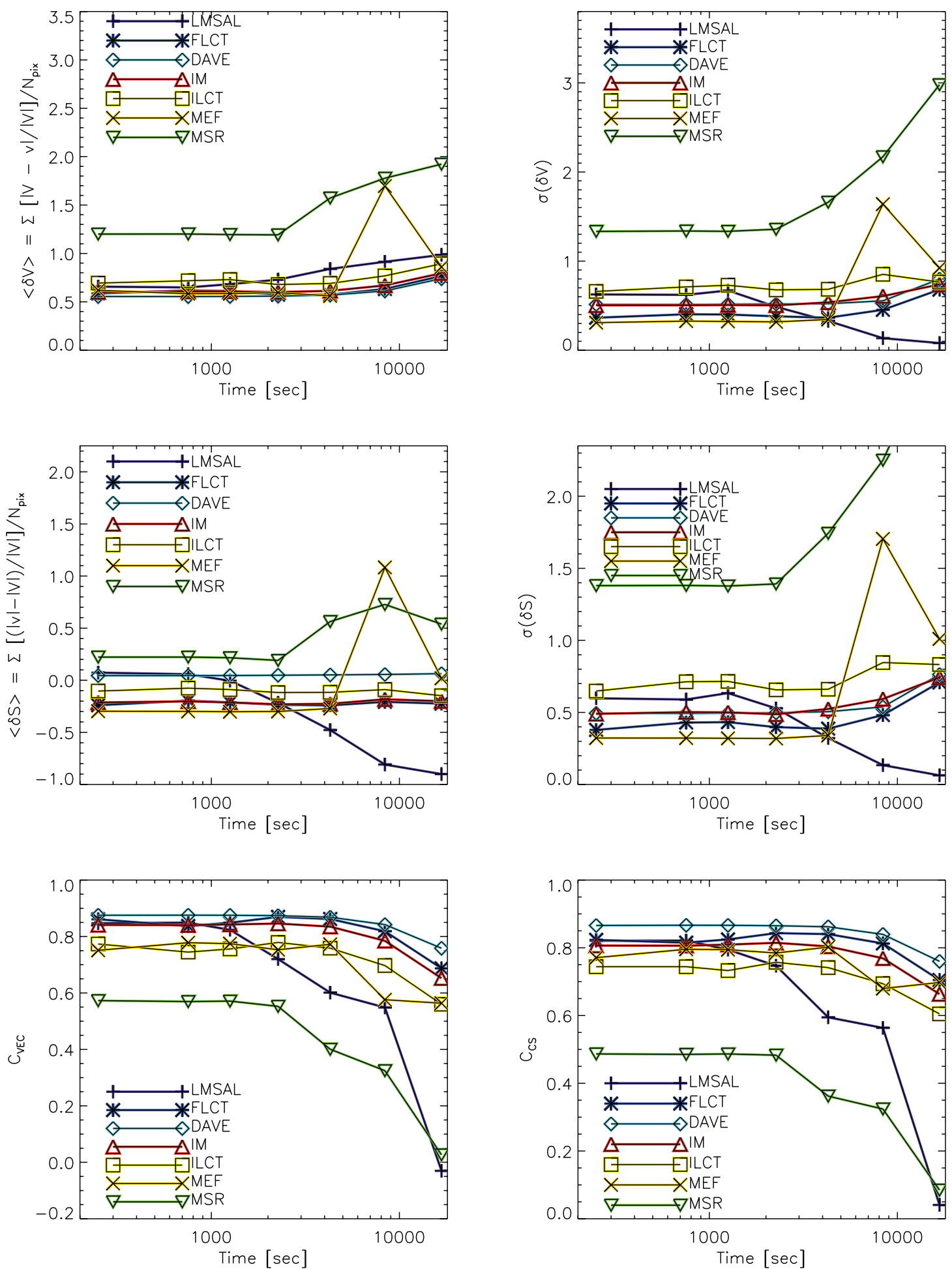

Fig. 8. -Each method's average fractional vector error (eq. [13]; top left) and the square root of its variance (eq. [14]; top right) as $\Delta t$ increases. Also shown are each method's average fractional error in speed (eq. [15]; middle left) and the square root of its variance (eq. [16]; middle right) as $\Delta t$ increases. We also plot each method's vector correlation $C_{\mathrm{vec}}$ (eq. [17]; bottom left) as $\Delta t$ increases. Finally, we plot each method's direction correlation $C_{\mathrm{CS}}$ (eq. [18]; bottom right) as $\Delta t$ increases.

MEF estimated the magnitude $\left|\boldsymbol{U} B_{z}\right|$ more accurately than $\left|\boldsymbol{V}_{\perp}\right|$ (in fact, MEF overestimated $\left|\boldsymbol{U} B_{z}\right|$, whereas it underestimated $\left.\left|\boldsymbol{V}_{\perp}\right|\right)$, and IM had an improved fractional vector error $(\sim 25 \%$ smaller) and vector correlation ( $40 \%$ higher).

\subsection{Comparisons of Other Quantities}

As discussed in the introduction, estimates of the components of velocity $\boldsymbol{v} \perp$ perpendicular to the magnetic field $\boldsymbol{B}$ can used to derive electric fields for driving MHD simulations, and to estimate the fluxes of magnetic energy and helicity across the surface imaged by the vector magnetograms used to derive $\boldsymbol{v}_{\perp}$. Accordingly, we compared estimates of these quantities with those from ANMHD.

All our methods assume ideal MHD governs the evolution of $B_{z}$. Under this assumption, the electric field $\boldsymbol{E}$ is derivable from $\boldsymbol{v}_{\perp}$ and $\boldsymbol{B}$ via $\boldsymbol{E}=-\left(\boldsymbol{v}_{\perp} \times \boldsymbol{B}\right) / c$. The induction equation implies 

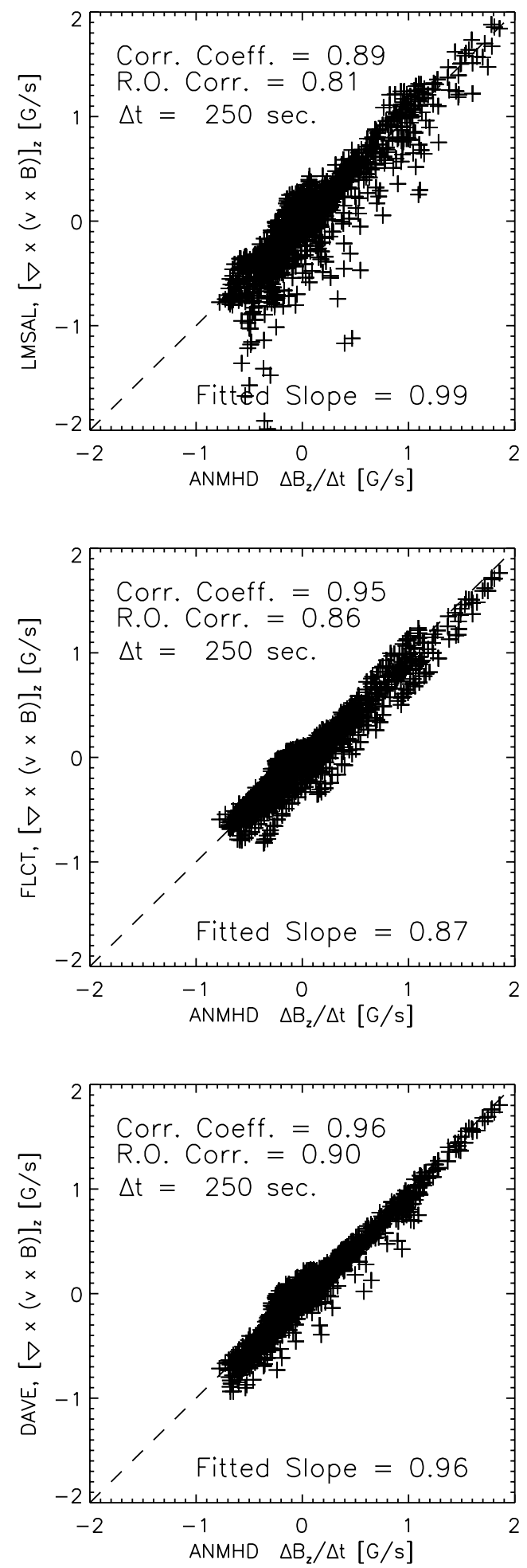

FIG. 9.-Pixel-by-pixel scatter plots of several methods' (LMSAL's LCT, FLCT, and DAVE; top to bottom $)$ estimates of $\left[\nabla \times\left(\boldsymbol{v}_{\perp} \times \boldsymbol{B}\right)\right] \cdot \hat{z}$ for $\Delta t=250 \mathrm{~s}$, which should match $\Delta B_{z} / \Delta t$ assuming ideal MHD.

that $\left[\nabla \times\left(\boldsymbol{v}_{\perp} \times \boldsymbol{B}\right)\right] \cdot \hat{z}$ should approximately match $\Delta B_{z} / \Delta t$, and Figures 9 and 10 show pixel-by-pixel scatter plots comparing these quantities, as well as the correlation coefficients and fitted slope (using a least-absolute-deviation method), for each technique. The scatter present in results from methods capable of deriving electric fields that match $\Delta B_{z} / \Delta t$ to machine accuracy (e.g., MEF, ILCT, and IM) can arise as an artifact of threshold masking applied to the velocity arrays prior to differentiating and/or regridding. Spatial derivatives were computed with a Fourier method, which introduced more scatter (and therefore decreased the correlation coefficients) than was present when finite-difference derivatives were used; but the fitted slope was better with the Fourier approach than with the finite-difference approach. (In addition, tests of both approaches with functions whose derivatives can be computed analytically showed the Fourier method to be more accurate when pixel-scale variations are present, as in real solar magnetograms.)

In Figures 11 and 12 we show pixel-by-pixel scatter plots comparing each method's estimated electric field components with ANMHD's, for $\Delta t=250 \mathrm{~s}$. Black forward slashes, dark-gray backslashes, and light-gray vertical bars correspond to $E_{x}, E_{y}$, and $E_{z}$, respectively. For each method, nonparametric Spearman rankorder correlation coefficients are also shown for each component of $\boldsymbol{E}$. All of our methods derive $\boldsymbol{v}_{\perp}$ from $\partial_{t} B_{z}$, which only depends on $E_{x}$ and $E_{y}$; hence, accurate reproduction of $E_{x}$ and $E_{y}$ is perhaps not surprising. But $E_{z} \propto-\left(v_{x} B_{y}-v_{y} B_{x}\right)$ is more accurately reconstructed, even though $v_{x} B_{y}$ and $v_{y} B_{x}$ do not directly affect $\partial_{t} B_{z}$. We note that $E_{z}$ plays a role in the evolution of the horizontal field, $\partial_{t} \boldsymbol{B}_{h}$, and must therefore be accurately specified to drive coronal MHD simulations in a manner consistent with the evolution of $\boldsymbol{B}_{h}$ observed in vector magnetograms. We address this point in more detail in $\S 5$. (For LMSAL's LCT, FLCT, and DAVE, we also tried using $\boldsymbol{u}$ instead of $\boldsymbol{v}_{\perp}$ to compute $\boldsymbol{E}$, and found no difference in the estimated electric field, as expected.)

The transport of magnetic energy across the photosphere is quantified by the normal component of the Poynting flux, $S_{z}=$ $c(\boldsymbol{E} \times \boldsymbol{B}) \cdot \hat{z} / 4 \pi$. Since magnetic energy powers flares and CMEs, quantifying the Poynting flux is important for understanding these phenomena. Assuming $\boldsymbol{E}=-\left(\boldsymbol{v}_{\perp} \times \boldsymbol{B}\right) / c$, the normal Poynting flux can be expressed in terms of either $\boldsymbol{v}_{\perp}$ or $\boldsymbol{u}$,

$$
S_{z}=\frac{\left(\boldsymbol{B}_{h} \cdot \boldsymbol{B}_{h}\right) v_{\perp, z}}{4 \pi}-\frac{\left(\boldsymbol{v}_{\perp, h} \cdot \boldsymbol{B}_{h}\right) B_{z}}{4 \pi}=-\frac{\boldsymbol{B}_{h} \cdot\left(\boldsymbol{u} B_{z}\right)}{4 \pi} .
$$

In Figures 13 and 14 we show pixel-by-pixel scatter plots comparing each method's estimated normal Poynting flux with ANMHD's, for $\Delta t=250 \mathrm{~s}$. The results shown were obtained using $\boldsymbol{v}_{\perp}$, but $\boldsymbol{u}$ was also tried for LMSAL's LCT, FLCT, and DAVE, and our results did not change.

For each method, the plots also list the linear (Pearson) and Spearman rank-order correlation coefficients. In addition, as a measure of the accuracy of the total estimated Poynting flux, we list the ratio of each method's integrated Poynting flux, $s_{z}$, to ANMHD's integrated Poynting flux, $S_{z}$,

$$
\text { ratio of totals } \equiv \frac{\sum s_{z}}{\sum S_{z}},
$$

where the sums run over the pixels for which the method estimated a nonzero velocity and for which $\left|B_{z}\right|$ exceeded $5 \%$ of $\max \left(\left|B_{z}\right|\right)$. Many of the methods fare relatively poorly at estimating the Poynting flux, although MEF, ILCT, and IM do show a significant correlation. MEF estimates ANMHD's total Poynting flux remarkably well, while IM and ILCT only recover $\sim 45 \%$ of the total. Both ILCT and IM proceeded from FLCT flows, which (from Fig. 8) systematically underestimate speeds; higher input speeds, from, e.g., DAVE, might give higher Poynting flux estimates. We note that FLCT's stronger correlation coefficients compared to those of DAVE do not improve its energy flux estimate; while FLCT might reproduce the trend of the ANMHD flux better, its systematically slow speed estimates limit its absolute accuracy. Scatter plots of the free energy flux (Welsch 2006) appear similar (not shown), with nearly identical correlation 

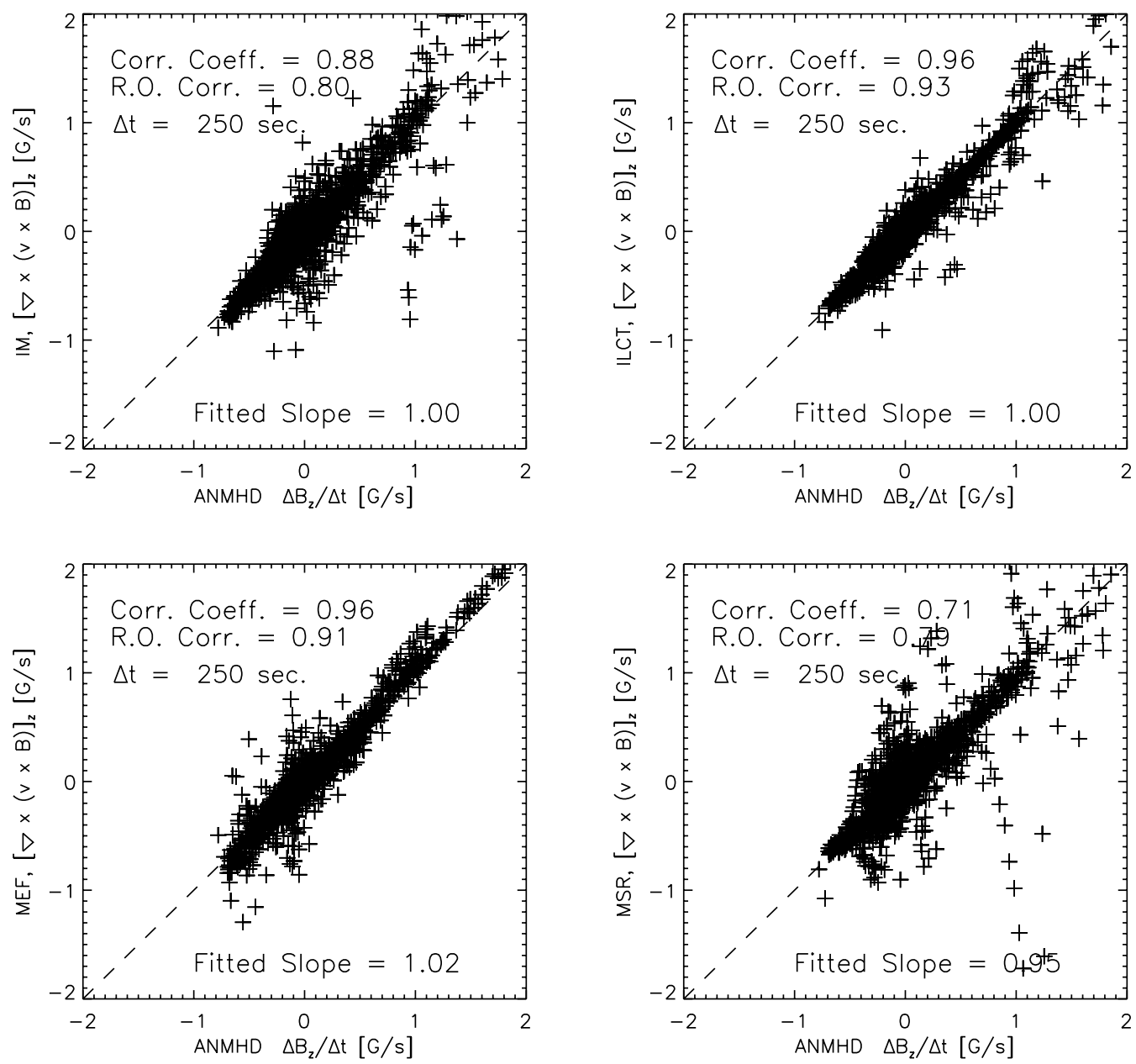

FIG. 10. - As with Fig. 9, but with the remaining methods' (IM, ILCT, MSR, and MEF; clockwise from top left) estimates of $\left[\nabla \times\left(\boldsymbol{v}_{\perp} \times \boldsymbol{B}\right)\right] \cdot \hat{z}$ vs. $\Delta B_{z} / \Delta t$.

coefficients; MEF, ILCT, and IM recovered similar fractions of the free energy flux. The orientations of the elongated clouds of estimates from ILCT and IM suggest that energy estimates could be improved by inflating these methods' estimates to empirically account for their observed biases. These results suggest that MEF, as well as modified versions of IM and ILCT, could be used to quantify the flux of magnetic energy into the corona. Further validation studies, using simulated magnetograms that more accurately represent photospheric evolution, should also be undertaken.

Finally, we also compare the net helicity flux from each method's estimated flows, $d h / d t$, with that from ANMHD's flows, $d H / d t$, for the shortest time interval, $\Delta t=250 \mathrm{~s}$. Because the methods of computing helicity fluxes by Berger \& Field (1984) and Pariat et al. (2005) are sensitive to different properties of the flow field, we computed the helicity flux both ways for our comparisons. We computed $d h_{\theta} / d t$ and $d H_{\theta} / d t$ after Pariat et al. (2005),

$$
\begin{aligned}
\frac{d H_{\theta}}{d t}= & -\frac{1}{2 \pi} \int d A B_{z}(\boldsymbol{x}) \\
& \times \int d A^{\prime} B_{z}\left(\boldsymbol{x}^{\prime}\right) \hat{z} \cdot\left\{\left(\boldsymbol{x}^{\prime}-\boldsymbol{x}\right) \times \frac{\left[\boldsymbol{U}\left(\boldsymbol{x}^{\prime}\right)-\boldsymbol{U}(\boldsymbol{x})\right]}{\left|\boldsymbol{x}^{\prime}-\boldsymbol{x}\right|}\right\},
\end{aligned}
$$

and similarly for $d h_{\theta} / d t$, with $\boldsymbol{U} \rightarrow \boldsymbol{u}$. Next we computed $d h_{A} / d t$ and $d H_{A} / d t$ after Berger \& Field (1984),

$$
d H_{A} / d t=2 \int d A\left[\left(\boldsymbol{B}_{h} \cdot \boldsymbol{A}_{P}\right) V_{z}-\left(\boldsymbol{V}_{\perp, h} \cdot \boldsymbol{A}_{P}\right) B_{z}\right]
$$

and similarly for $d h_{A} / d t$, with $\boldsymbol{V}_{\perp} \rightarrow \boldsymbol{v}_{\perp}$, and where $\hat{z} \cdot \nabla_{h} \times \boldsymbol{A}_{P}=$ $B_{z}$ and $\nabla_{h} \cdot \boldsymbol{A}_{P}=\hat{\boldsymbol{z}} \cdot \boldsymbol{A}_{P}=0$.

The results from these approaches are shown in Table 1. Using either formalism, MEF matches ANMHD's helicity flux very accurately. ILCT and IM both substantially underestimate the helicity flux, but do a better job than the remaining methods, which do significantly worse, even getting the sign wrong in some cases. As with the Poynting flux estimates for ILCT and IM, the lowspeed bias of the FLCT input flows might partly explain their low estimates; higher input speeds, from, e.g., DAVE, might give higher helicity flux estimates (see also Chae 2007). The referee noted that the methods that estimated $\boldsymbol{U} B_{z}$ more accurately also estimated $d H / d t$ more accurately. The tracking methods, LMSAL's LCT, FLCT, and DAVE, did better at estimating the helicity flux via equation (23) than via equation (22). We note that the MEF velocities were estimated over a smaller region of the test data set than the other methods; the region excluded by MEF lies at the lower left of the field of view in Figure 5 (bottom left). Since, however, most methods performed well in most of this region, we believe the bias introduced by omitting MEF flow estimates in this region to be slight. The first column of Table 1 gives the number of pixels used in this helicity comparison. (The same pixels were used for all comparisons from the shortest time interval.) The results for longer time intervals (not shown) were similar.

One possible explanation for most methods' poor performance at recovering the helicity flux is that the helicity flux could be relatively small. This is consistent with the facts that the rising 

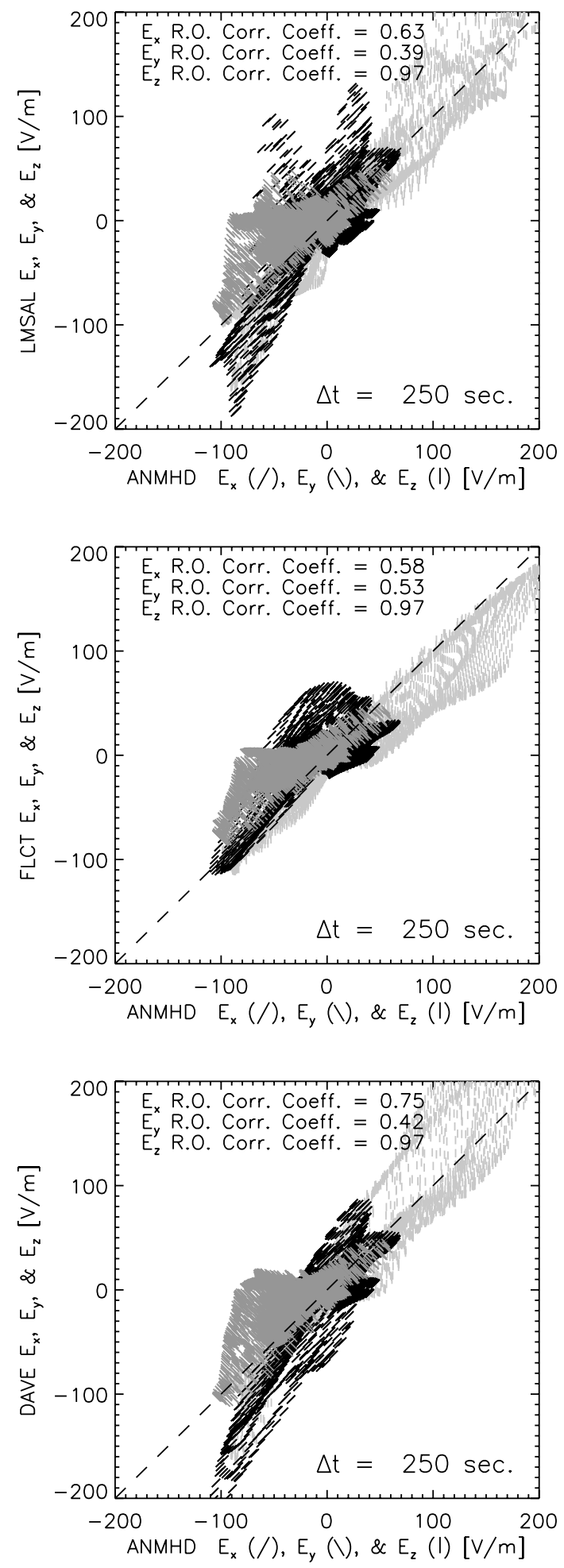

Fig. 11.-Pixel-by-pixel scatter plots comparing several methods' (LMSAL's LCT, FLCT, and DAVE; top to bottom) estimated electric field components with ANMHD's, for $\Delta t=250 \mathrm{~s}$. Black forward slashes, dark-gray backslashes, and lightgray vertical bars correspond to $E_{x}, E_{y}$, and $E_{z}$, respectively. For each method, nonparametric Spearman rank-order correlation coefficients are also shown for each component of $\boldsymbol{E}$.

flux tube was initially untwisted and that the convecting background plasma did not include the effects of rotation, which might introduce twist (Longcope et al. 1998). This explanation, however, does not obviously explain most methods' similarly poor performance at recovering the Poynting flux. The referee pointed out that the ratio of the helicity flux to the square of magnetic flux, a characteristic measure of the rate of helicity injection, is similar to that reported for solar observations, on the order of $10^{-6} \mathrm{~s}^{-1}$ (Pariat et al. 2006), which suggests the helicity injection rate is not exceptionally low. Nonetheless, comparative studies with a highly twisted, rising flux tube would be worthwhile.

As an aside, we found that computing the vector potential, $\boldsymbol{A}_{P}$, via the Fourier approach of Chae (2001) resulted in differences of $\sim 10 \%$ in the helicity flux compared to that calculated using either the Berger \& Field (1984) approach with a Green's function scheme to compute $\boldsymbol{A}_{P}$, or the approach of Pariat et al. (2005). In addition, if $\boldsymbol{A}_{P}$ was computed by Fourier and Green's function methods over a subregion of the full simulation domain, the differences between the two resulting helicity fluxes increased. It appears that the assumption of periodicity can increase the magnitude of $\boldsymbol{A}_{P}$, even far from regions of strong $B_{z}$, which affects $d H / d t$. These points are also discussed by Chae (2007).

\section{DISCUSSION AND CONCLUSIONS}

Using time series of synthetic magnetograms extracted from MHD simulations, in which the magnetic and velocity fields are known, we used several techniques to estimate the velocity field solely from the evolution of the magnetic field. We then used several approaches to characterize the accuracy of the estimated velocities, as well as quantities derivable from such estimates: electric fields and the fluxes of magnetic energy and helicity.

Which velocity-inversion method is superior? In tests performed thus far, no method works as well as we would like; every method exhibited significant errors. By many measures, velocities determined by FLCT, DAVE, IM, ILCT, and MEF performed comparably in estimating ANMHD's velocities. LMSAL's LCT performed well when typical shifts were smaller than 1 pixel, but performed poorly when typical shifts exceeded a pixel. Consistently, MSR did not perform as well as the other methods.

Reviewing performance metrics separately is illuminating. In terms of the mean vector error, all methods did poorly, but the best, DAVE, FLCT, and MEF, performed comparably. DAVE was marginally more accurate in direction and magnitude than other methods, on average, but the differences were not statistically significant. MEF was marginally more accurate at determining the electric field, but recovered the fluxes of magnetic energy (the Poynting flux) and magnetic helicity essentially perfectly. Hence, we conclude that, while its performance was far from perfect, MEF performed better in our analyses than the other methods tested.

We address the possibility that the physical assumption used by MEF is more suited than those of the other approaches we tested to the particular physical assumptions employed in the ANMHD simulations. Specifically, the action in the Lagrangian formalism that can used to derive the equations solved by ANMHD contains spatial integrals over the product of the density and the velocity squared, a term similar to that minimized by MEF (the integral of the velocity squared over a constant $z$ layer). The mean and median of the ratio of magnetic energy density to kinetic energy density in pixels with $\left|B_{z}\right|>5 \%$ of $\max \left(\left|B_{z}\right|\right)$ are 0.19 and 0.13 , respectively, meaning the magnetic energy term in the Lagrangian is not dominant, and could perhaps be neglected. MEF does, in fact, ignore magnetic forces and energy, only using the normal field evolution and magnetic geometry as constraints on the velocity minimization. The hydrodynamic and gravitational terms in the full Lagrangian, however, describe physical effects that are not treated by MEF, and which cannot be neglected in the anelastic formalism. Consequently, we conclude that the resemblance between the term minimized by MEF and the kinetic term in the Lagrangian that forms a basis for the anelastic formalism cannot 

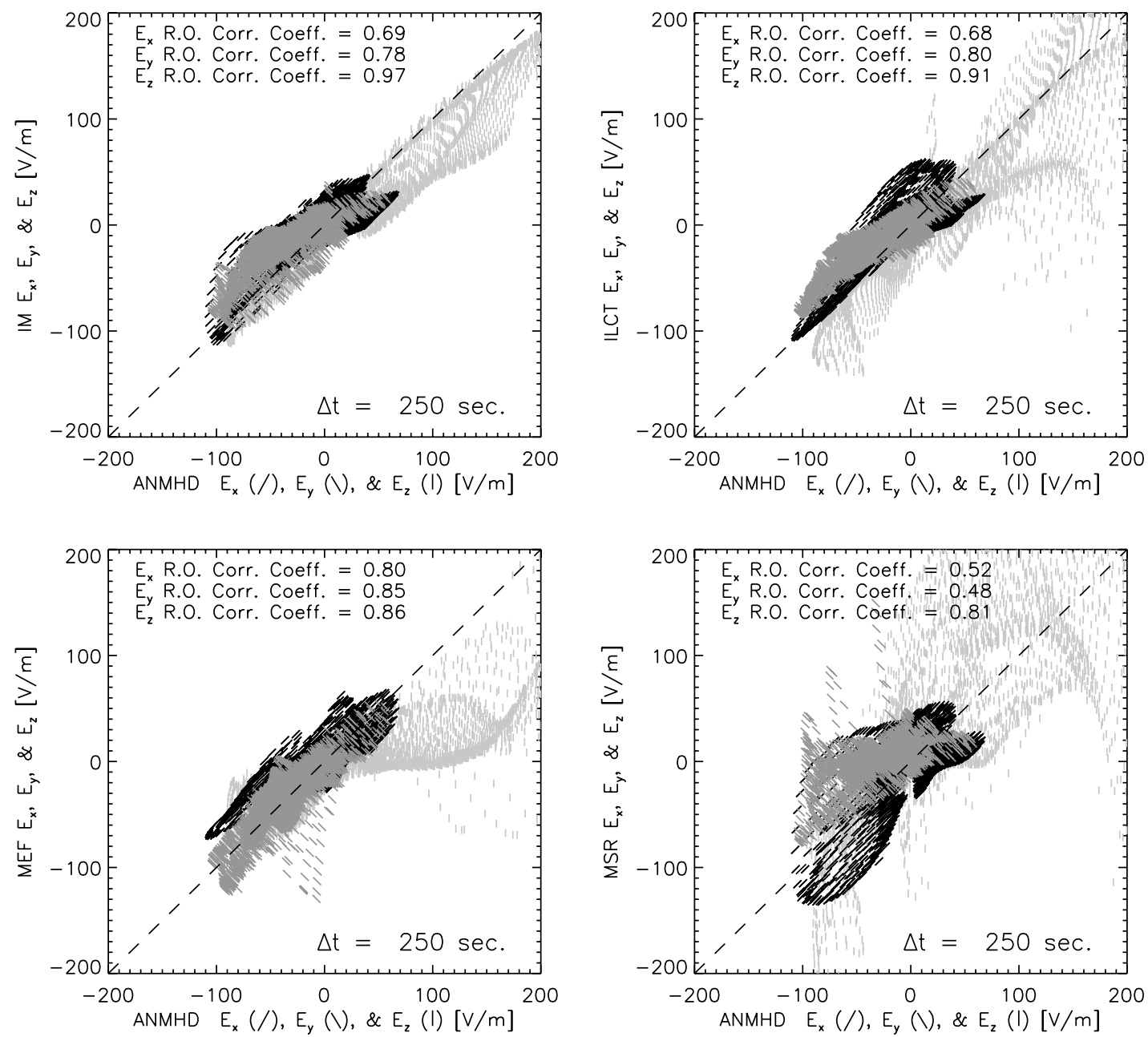

FIG. 12.-As with Fig. 11, but with the remaining methods' (IM, ILCT, MSR, and MEF; clockwise from top left) electric field estimates.

fully explain MEF's superior results at computing the Poynting and helicity fluxes, although it might partly explain them.

How might our techniques be improved? Assumptions made in the implementation of some of our techniques could be modified. For instance, the 1 pixel cap on allowed displacements assumed by LMSAL's LCT technique could be overridden for application to images separated by a $\Delta t$ longer than the pixel crossing time. Homogeneous boundary conditions on one of ILCT's scalar potentials, $\chi$ or $\psi$, at the edges of tracked pixels (defined by the threshold in $\left|B_{z}\right|$ prior to tracking) might improve velocity estimates near these edges.

We note that the "inductive" corrections added to the FLCT flows by ILCT and IM led to significantly improved estimates of the fluxes of magnetic energy and helicity. This recommends an approach that combines results from an optical flow technique, like FLCT, LMSAL's LCT, or DAVE, with an inductive technique, like MEF, ILCT, or IM.

Would the methods tested here perform similarly in tests with other simulated data? Gibson et al. (2004) also used FLCT to attempt reconstructing flows in an emerging flux rope simulation. They found that FLCT did a poor job of reconstructing the actual flows, and that the helicity flux inferred from the FLCT results was substantially lower than the actual helicity flux. Although the ANMHD simulations used exhibit much more spatial structure than the more idealized simulation of Gibson et al. (2004), many methods we tested still failed to reproduce the actual helicity flux. Gibson et al. (2004) did not quantitatively compare the estimated and actual flow fields, which precludes a direct comparison of the accuracy of FLCT between the idealized simulations of Gibson et al. (2004) and the more realistic simulations used here.

As a tool for testing velocity-inversion techniques, however, the ANMHD data set used in our tests does have shortcomings. As pointed out in $\S 3.7$, the model's flows do not occur in a layer with a strong upward, negative density gradient, as in the photosphere. In addition, simple "moving paint" experiments, in which we shifted a pair of images with respect to one another by displacements $\delta x$ and $\delta y$ that were constant in space, revealed that some flow-inversion techniques behave significantly worse with synthetic ANMHD magnetograms than with actual magnetograms. For instance, using several shifts, with both integer-pixel and fractional-pixel displacements (with cubic convolution interpolation for the latter), we found that all measures of error were larger with ANMHD data than with a vector magnetogram from AR 8210 used in an earlier study by Welsch et al. (2004). Quantitative results from a test with a fractional shift of $(\delta x, \delta y)=(0.2,0.7)$ are shown in Table 2, along with results for FLCT extracted from Figure 8 , for $\Delta t=250 \mathrm{~s}$. Clearly, FLCT reconstructs constant shifts applied to ANMHD data more accurately than an actual ANMHD flow field, but FLCT does a much better job at reproducing constant shifts applied to a real magnetogram. This 

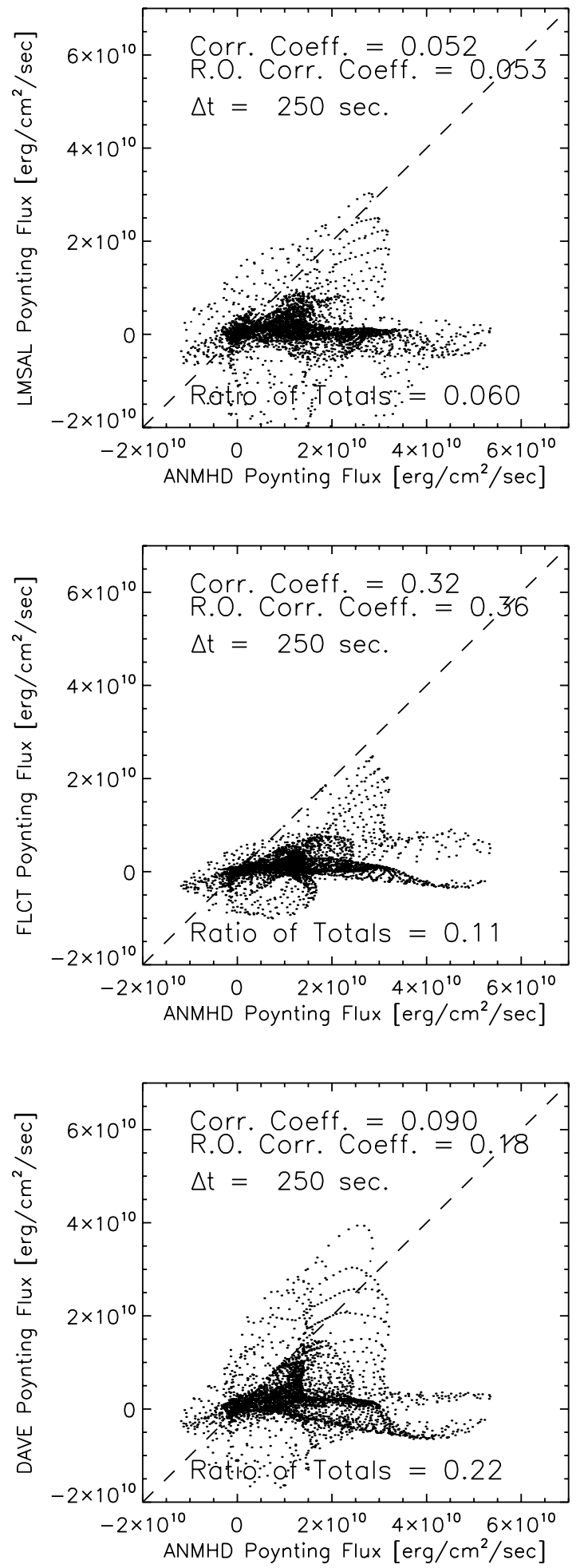

FIG. 13.-Pixel-by-pixel scatter plots comparing several methods' (LMSAL's LCT, FLCT, and DAVE; top to bottom) estimated normal Poynting flux with ANMHD's, for $\Delta t=250 \mathrm{~s}$. For each method, linear and rank-order correlation coefficients are shown, as is the ratio of the integrated estimated Poynting flux to the integrated actual Poynting flux.

simple test suggests that the ANMHD data set either lacks some characteristic present in real solar magnetograms or contains artifacts not present in solar data. One possibility is that the Sun's photospheric field exhibits spatial variations on scales not reproduced in the ANMHD simulations; the ANMHD fields do vary smoothly in space compared to solar observations at similar resolution, such as MDI high-resolution images (with $\sim 450 \mathrm{~km}$ pixels) of activeregion fields (e.g., Ravindra 2006). We note that ANMHD uses dealiasing for nonlinear terms, which keeps power in the upper third of wavenumbers at zero. This essentially imposes a degree of smoothing: short-wavelength magnetic field variations are not modeled. Another possibility is that Fourier ringing present in strong fields in the ANMHD data set, mentioned briefly in $\S 2$, adversely affects the performance of some of our velocity estimation methods.

Despite its shortcomings, the ANMHD data set that we used was the best available at the time. Realistic tests must involve upflows and downflows; hence, moving paint experiments (even with spatially varying shifts) are too simplistic. Other available simulations of photospheric flux evolution either modeled field evolution on small scales (Stein \& Nordlund 2006; Bercik 2002) or lacked both convection-dominated velocity fields and magnetic fields varying on small spatial scales (Gibson et al. 2004; Manchester et al. 2004; Magara 2004).

To assess the performance of our methods with simulated data that are more realistic, development of more sophisticated models for future tests is ongoing. Soon we hope to conduct tests using magnetograms extracted from theoretical simulations using the RADMHD code (Abbett 2007), which treats the photospheric layers more realistically than ANMHD can. We also plan to conduct related tests with data-driven simulations (Abbett et al. 2004) using time series of high-resolution vector magnetograms from SOT on Hinode, when these data become available. Assuming synthetic data sets from these more sophisticated simulations exhibit structure on smaller spatial scales, it is possible that some of the methods tested here will perform relatively better.

The methods analyzed here were developed to determine flow fields consistent with the evolution of $B_{z}$. Hence, these flows are not necessarily consistent with the evolution of $\boldsymbol{B}_{h}$, although most methods did well at estimating $E_{z}$ (which partially governs $\partial_{t} \boldsymbol{B}_{h}$; see eq. [1]), as shown in Figures 11 and 12. As Démoulin \& Berger (2003) observed, there exists a class offlows that cause no evolution in $B_{z}$ but that can inject large amounts of magnetic energy and helicity. (Flows along contours of $B_{z}$ ["contour flows"] are well-known members of this set, but modelers have employed other flows that do not alter $B_{z}$; see, e.g., Lynch et al. 2005.) Any velocity estimation method that depends on $\Delta B_{z} / \Delta t$ alone will, however, be insensitive to such flows. Our results indicate that tracking methods (LMSAL's LCT, FLCT, and DAVE) are particularly insensitive to the injection of energy and helicity. Changes in magnetic energy and helicity should, however, lead to evolution in the measured horizontal field, $\partial_{t} \boldsymbol{B}_{h}$, meaning velocity estimation methods that incorporate $\partial_{t} \boldsymbol{B}_{h}$ could be more sensitive to such motions. In addition, for driving dynamic coronal models using sequences of photospheric and/or chromospheric vector magnetograms, the driving flows must be consistent with the observed evolution of the three-component magnetic vector in the magnetogram layer(s). Because the temporal evolution of the horizontal components of $\boldsymbol{B}$ depends, via the induction equation, on vertical gradients in the components of both the magnetic and velocity fields, the problem is fundamentally three-dimensional. Hence, velocities must be estimated in a layer offinite thickness, not just in a two-dimensional plane, as with the method studied here. Extension of existing techniques to this more challenging problem is an ongoing area of research.

We note that Doppler measurements of the velocity of the magnetized plasma (e.g., Chae et al. 2004) do not, in general, fully determine plasma flows either along or perpendicular to the magnetic field. Doppler measurements can, however, be combined with one of the velocity estimation techniques tested here - which, 

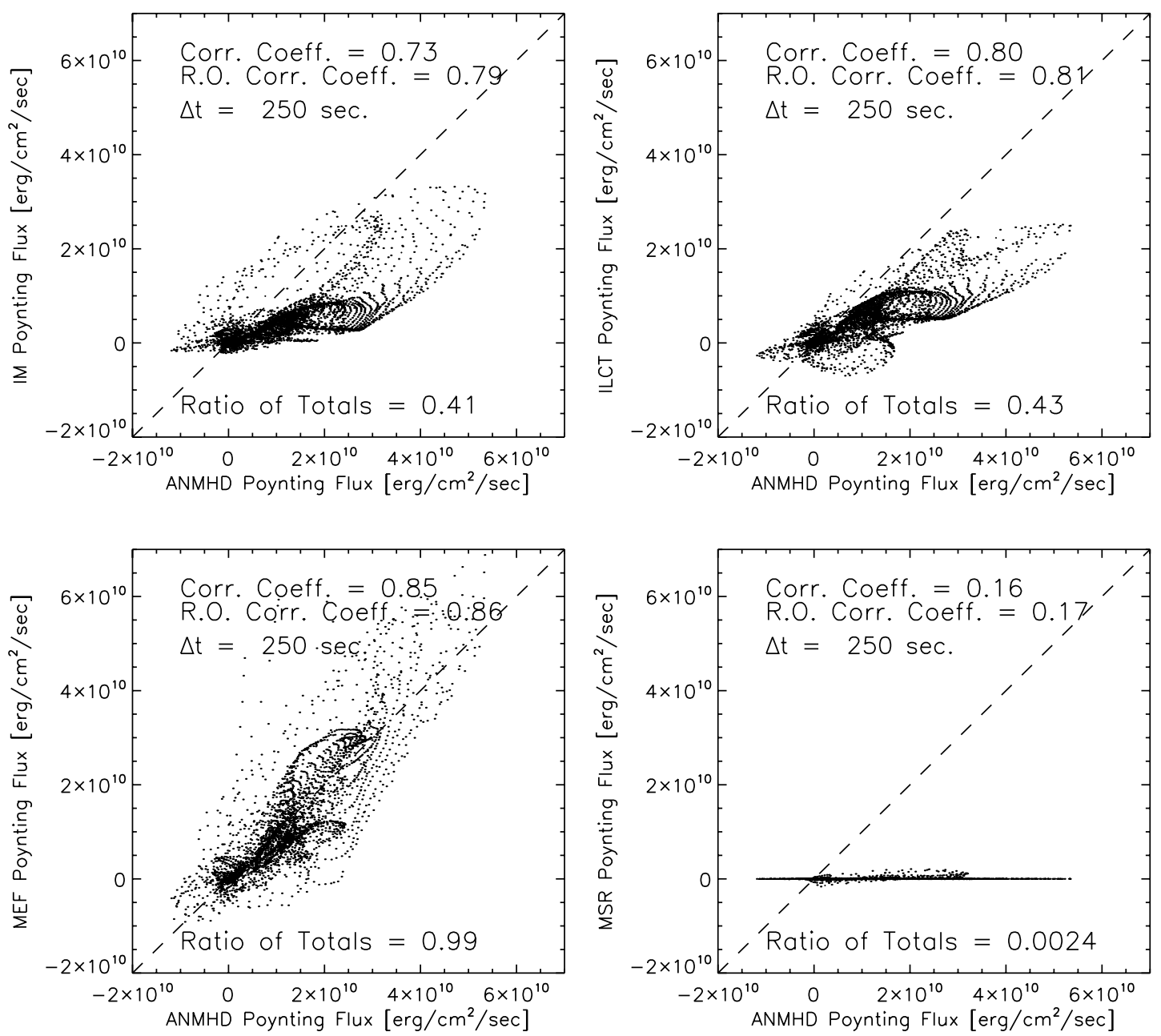

FIG. 14.-As with Fig. 13, but with the remaining methods' (IM, ILCT, MSR, and MEF; clockwise from top left) Poynting flux estimates.

TABLE 1

COMPARISONS OF $d H / d t$

\begin{tabular}{|c|c|c|c|c|c|c|}
\hline Method & $\begin{array}{c}N \\
\text { (pixels) }\end{array}$ & $\begin{array}{c}d H_{\theta} / d t^{\mathrm{a}} \\
\left(\mathrm{Mx}^{2} \mathrm{~s}^{-1}\right)\end{array}$ & $\begin{array}{c}d h_{\theta} / d t \\
\left(\mathrm{Mx}^{2} \mathrm{~s}^{-1}\right)\end{array}$ & $d h_{\theta} / d H_{\theta}$ & $\begin{array}{c}d h_{A} / d t \\
\left(\mathrm{Mx}^{2} \mathrm{~s}^{-1}\right)\end{array}$ & $d h_{A} / d H_{A}$ \\
\hline LMSAL.. & 4194 & $-3.23 E+37$ & $2.21 \mathrm{E}+36$ & -0.07 & $-3.59 \mathrm{E}+36$ & 0.11 \\
\hline FLCT ........................ & 4195 & $-3.23 E+37$ & $2.24 \mathrm{E}+36$ & -0.07 & $-2.81 \mathrm{E}+36$ & 0.09 \\
\hline DAVE ........................ & 4169 & $-3.23 E+37$ & $3.21 \mathrm{E}+36$ & -0.10 & $-7.50 \mathrm{E}+36$ & 0.23 \\
\hline 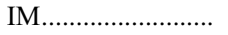 & 4195 & $-3.23 \mathrm{E}+37$ & $-9.00 \mathrm{E}+36$ & 0.28 & $-8.15 \mathrm{E}+36$ & 0.25 \\
\hline ILCT.......................... & 4195 & $-3.23 \mathrm{E}+37$ & $-1.18 \mathrm{E}+37$ & 0.37 & $-1.18 \mathrm{E}+37$ & 0.36 \\
\hline MEF ……................... & 3762 & $-3.21 \mathrm{E}+37^{\mathrm{b}}$ & $-3.19 \mathrm{E}+37$ & 1.00 & $-3.24 \mathrm{E}+37$ & 0.99 \\
\hline MSR …...................... & 4127 & $-3.23 E+37$ & $1.17 \mathrm{E}+37$ & -0.36 & $1.17 \mathrm{E}+37$ & -0.36 \\
\hline
\end{tabular}

${ }^{\text {a }}$ Except where noted, $d H_{A} / d t=d H_{\theta} / d t$.

${ }^{\mathrm{b}}$ Here $d H_{A} / d t=-3.24 \times 10^{37}$. 
TABLE 2

Comparisons of FlCT Performance with Various Data

\begin{tabular}{ccccc}
\hline \hline Data Set & $\left\langle\delta \boldsymbol{V}_{\perp}\right\rangle \pm \sigma\left(\delta \boldsymbol{V}_{\perp}\right)^{\mathrm{a}}$ & $\langle\delta S\rangle \pm \sigma(\delta S)^{\mathrm{b}}$ & $C_{\mathrm{vec}}{ }^{\mathrm{c}}$ & $C_{\mathrm{CS}}{ }^{\mathrm{d}}$ \\
\hline ANMHD $^{\mathrm{e}} \ldots \ldots \ldots \ldots . . . . . . .$. & $0.59 \pm 0.36$ & $-0.24 \pm 0.38$ & 0.82 & 0.86 \\
ANMHD, shifted ........ & $0.34 \pm 0.17$ & $-0.28 \pm 0.22$ & 0.9 & 1.0 \\
AR 8210, shifted......... & $0.10 \pm 0.04$ & $-0.04 \pm 0.07$ & 1.0 & 1.0 \\
\hline
\end{tabular}

${ }^{\text {a }}$ Eqs. (13) and (14).

b Eqs. (15) and (16).

c Eq. (17).

d Eq. (18).

e Extracted from Fig. 8 ( $(2)$, for $\Delta t=250 \mathrm{~s}$.

being derived from $\partial_{t} B_{z}$, only depend on the components of the flow perpendicular to $\boldsymbol{B}$, as explained above - to recover the complete velocity vector, i.e., components both parallel and perpendicular to the magnetic field. First, time series of magnetograms can be used to infer the component of the velocity $\boldsymbol{v}_{\perp}$ perpendicular to $\boldsymbol{B}$, using one of the methods presented here. Then, the projection of this velocity along the LOS direction, $\hat{\ell}$, can be subtracted from the Doppler velocity to give the line-of-sight component of $v_{\|}$, the component of the velocity parallel to $\boldsymbol{B}$. By dividing this line-of-sight component of $v_{\|}$by the cosine of the angle between $\boldsymbol{B}$ and $\hat{\ell}, v_{\|}$can be found. Georgoulis \& LaBonte
(2006) have employed this approach in an observational study of active-region flows.

We all thank the anonymous referee, whose careful reading and comprehensive report significantly improved the paper. W. P. A., G. H. F., D. W. L., B. R., and B. T. W. acknowledge the Department of Defense MURI grant, "Understanding Solar Eruptions and Their Interplanetary Consequences." W. P. A., G. H. F., and B. T. W. also acknowledge support from the NSF via the SHINE program under ATM 03-27712a, as well as from grant NNG 05-G144G-04/08 from NASA's Sun-Earth Connections Theory Program. M. K. G. acknowledges partial support for this work by NASA grant NNG $05-G M 47 G$. K. K. acknowledges Grant-in-Aid for Creative Scientific Research "The Basic Study of Space Weather Prediction" (17GS0208; head investigator: K. Shibata) from the Ministry of Education, Science, Sports, Technology, and Culture of Japan, as well as Grant-in-Aid for Scientific Research (B) (19340180; head investigator: K. Kusano) from the Japan Society for the Promotion of Science. P. W. S. acknowledges support from NASA's Living with a Star program, under grant TR\&T-NNH05ZDA001N. B. T. W. appreciates the hard work of all co-authors, and thanks Pascal Démoulin, Mitch Berger, and Etienne Pariat, as well as Maureen Lahiff, for stimulating discussions that improved the manuscript.
Abbett, W. P. 2007, ApJ, 665, 1469

Abbett, W., Fisher, G., \& Fan, Y. 2000, ApJ, 540, 548

Abbett, W. P., Fisher, G. H., Fan, Y., \& Bercik, D. J. 2004, ApJ, 612, 557

Amari, T., Luciani, J. F., Mikic, Z., \& Linker, J. 2000, ApJ, 529, L49

Antiochos, S. K., DeVore, C. R., \& Klimchuk, J. A. 1999, ApJ, 510, 485

Barnes, G., Longcope, D. W., \& Leka, K. D. 2005, ApJ, 629, 561

Bercik, D. J. 2002, Ph.D. thesis, Michigan State Univ.

Berger, M. A., \& Field, G. B. 1984, J. Fluid Mech., 147, 133

Chae, J. 2001, ApJ, 560, L95

2007, Adv. Space Res., 39, 1700

Chae, J., Moon, Y., \& Pevtsov, A. A. 2004, ApJ, 602, L65

Démoulin, P., \& Berger, M. A. 2003, Sol. Phys., 215, 203

DeRosa, M. L. 2001, Ph.D. thesis, Univ. Colorado

Fan, Y., Abbett, W. P., \& Fisher, G. H. 2003, ApJ, 582, 1206

Fan, Y., Zweibel, E. G., Linton, M. G., \& Fisher, G. H. 1999, ApJ, 521, 460

Fermuller, C. 2001, Comput. Vision Image Understanding, 82, 1

Fisher, G. H. 2006, BAAS SPD Meeting, 37, 03.02

Forbes, T. G. 2000, J. Geophys. Res., 105, 23153

Georgoulis, M. K., \& LaBonte, B. J. 2006, ApJ, 636, 475

Georgoulis, M. K., LaBonte, B. J., \& Metcalf, T. R. 2004, ApJ, 602, 446

Gibson, S. E., Fan, Y., Mandrini, C., Fisher, G., \& Démoulin, P. 2004, ApJ, 617,600

Gosling, J. T. 1993, J. Geophys. Res., 98, 18937

Henney, C. J., et al. 2002, BAAS, 200, 55.14

Hurlburt, N. E., Schrijver, C. J., Shine, R. A., \& Title, A. M. 1995, in Helioseismology, ed. J. T. Hoeksema et al. (ESA SP-376; Noordwijk: ESA), 239

Kusano, K., Maeshiro, T., Yokoyama, T., \& Sakurai, T. 2002, ApJ, 577, 501

. 2004, in ASP Conf. Ser. 325, The Solar-B Mission and the Forefront

of Solar Physics, ed. T. Sakurai \& T. Sekii (San Francisco: ASP), 175

Lantz, S. R., \& Fan, Y. 1999, ApJS, 121, 247

Leka, K. D., \& Barnes, G. 2003a, ApJ, 595, 1277

$$
\text { 2003b, ApJ, 595, } 1296
$$$$
\text { 2007, ApJ, 656, } 1173
$$

Leka, K. D., \& Metcalf, T. R. 2003, Sol. Phys., 212, 361

Lionello, R., Mikić, Z., Linker, J. A., \& Amari, T. 2002, ApJ, 581, 718

Longcope, D. W. 2004, ApJ, 612, 1181

\section{REFERENCES}

Longcope, D. W., Fisher, G. H., \& Pevtsov, A. A. 1998, ApJ, 507, 417

Lucas, B. D., \& Kanade, T. 1981, in Proc. 7th International Joint Conference on Artificial Intelligence, ed. J. P. Hayes (Los Altos: Kaufmann), 121

Luhmann, J. G., Gosling, J. T., Hoeksema, J. T., \& Zhao, X. 1998, J. Geophys. Res., 103, 6585

Lynch, B. J., et al. 2005, in Connecting Sun and Heliosphere (ESA SP-592; Noordwijk: ESA), 16

Maeshiro, T., Kusano, K., Yokoyama, T., \& Sakurai, T. 2005, ApJ, 620, 1069 Magara, T. 2004, ApJ, 605, 480

Manchester, W., IV, Gombosi, T., DeZeeuw, D., \& Fan, Y. 2004, ApJ, 610, 588

Metcalf, T. R., Leka, K. D., \& Mickey, D. L. 2005, ApJ, 623, L53

Moon, Y.-J., Chae, J., Wang, H., Choe, G. S., \& Park, Y. D. 2002, ApJ, 580, 528

November, L., \& Simon, G. 1988, ApJ, 333, 427

Pariat, E., Aulanier, G., Schmieder, B., Georgoulis, M. K., Rust, D. M., \& Bernasconi, P. N. 2004, ApJ, 614, 1099

Pariat, E., Démoulin, P., \& Berger, M. A. 2005, A\&A, 439, 1191

Pariat, E., Nindos, A., Démoulin, P., \& Berger, M. A. 2006, A\&A, 452, 623

Press, W. H., Teukolsky, S. A., Vetterling, W. T., \& Flannery, B. P. 1992, Numerical Recipes in C: The Art of Scientific Computing (2nd ed.; Cambridge: Cambridge Univ. Press)

Ravindra, B. 2006, Sol. Phys., 237, 297

Régnier, S., Canfield, R. C., \& Longcope, D. W. 2006, A\&A, 451, 319

Roussev, I. I., Sokolov, I. V., Forbes, T. G., Gombosi, T. I., Lee, M. A., \& Sakai, J. I. 2004, ApJ, 605, L73

Scherrer, P., et al. 2005, AGU Spring Meeting, SP43A-05

Schrijver, C. J., et al. 2006, Sol. Phys., 235, 161

Schuck, P. W. 2005, ApJ, 632, L53 2006, ApJ, 646, 1358

Stein, R. F., \& Nordlund, ̊̊. 2006, ApJ, 642, 1246

Tarbell, T. D. 2006, BAAS SPD Meeting, 37, 36.02

Title, A. M., Hurlburt, N. E., Schrijver, C. J., Shine, R. A., \& Tarbell, T. 1995, in Helioseismology, ed. J. T. Hoeksema et al. (ESA SP-376; Noordwijk: ESA), 113

Welsch, B. T. 2006, ApJ, 638, 1101

Welsch, B. T., Fisher, G., \& Abbett, W. 2004, ApJ, 610, 1148 